\title{
AN ANALYSIS OF THE COBWEB MODEL WITH BOUNDEDLY RATIONAL HETEROGENEOUS PRODUCERS
}

\author{
CARL CHIARELLA* ${ }^{*}$ XUE-ZHONG HE*† AND PEIYUAN ZHU\# \\ * SCHOOL OF FINANCE AND ECONOMICS \\ UNIVERSITY OF TECHNOLOGY, SYDNEY \\ PO BOX 123, BROADWAY NSW 2007, AUSTRALIA \\ \# DEPARTMENT OF ELECTRICAL AND INFORMATION ENGINEERING \\ THE UNIVERSITY OF SYDNEY \\ NSW 2006, AUSTRALIA
}

SEPTEMBER 25, 2003

\begin{abstract}
This paper studies the dynamics of the traditional cobweb model with risk averse heterogeneous producers who seek to learn the distribution of asset prices using geometric decay processes (GDP) — the expected mean and variance are estimated as a geometric weighted average of past observations-with either finite or infinite fading memory. With constant absolute risk aversion, the dynamics of the nonlinear model can be characterized with respect to the length of memory window and the memory decay rate of the learning GDP. The dynamics of such heterogeneous learning processes and the capacity of the producers to learn are discussed. It is found that the interaction of heterogeneity, risk aversion, memory decay rate and window length of the GDP play a complicated role on the price dynamics of the nonlinear cobweb model. In general, an increase of the memory decay rate plays a stabilizing role on the local stability of the steady state price when the memory length is infinite, but this role becomes ambiguous when the memory is finite.
\end{abstract}

JEL classifications: D83; D84; E21; E32, C60

Keywords: Cobweb model, heterogeneity, bounded rationality, geometric decay learning dynamics, bifurcations

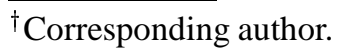




\section{INTRODUCTION}

Recent years have seen a great deal of research into the dynamics of economies populated by boundedly rational heterogeneous agents (e.g. Day and Huang (1990)), Brock and Hommes (1997)). In this paper, we introduce boundedly rational heterogeneous agents into the traditional cobweb model. In particular, we analyze the interaction of heterogeneity, agents' risk aversion and their attempts to learn from past time series of prices. We show that, on the one hand, heterogeneous learning can help agents to learn the rational equilibrium in some cases, on the other hand, such learning can lead to market instability and to periodic, or even chaotic, price fluctuations.

For the well-known cobweb model with linear supply $p_{t}^{e}=a q_{t}+b$ and demand $p_{t}=\alpha q_{t}+\mu$, where, $q_{t}$ and $p_{t}$ are quantities and prices, respectively, at period $t, p_{t}^{e}$ is the price expected at time $t$ based on the information at $t-1$, and $a, b, \mu(>0)$ and $\alpha<0$ are constants, it is well known that, under the naive expectation scheme $p_{t}^{e}=p_{t-1}$, the price either converges to the market equilibrium (when $|\alpha / a|<1$ ) or explodes (when $|\alpha / a|>1$ ). To obtain more realistic, bounded oscillatory, price time paths, the literature has introduced non-linearities into the cobweb model. Such nonlinearities can come from either nonlinear supply or demand curves, risk aversion (discussed in what follows), or agents' heterogeneity, bounded rationality and various learning processes.

When the producers are homogeneous, it has been shown that agents' expectations and non-linearities in the supply or demand curves may lead the cobweb model to exhibit both stable periodic and chaotic behavior (e.g., Artsein (1983), Jensen and Urban (1984), Chiarella (1988), Holmes and Manning (1988), Hommes (1991, 1994, 1998), Puu (1991) and Day (1992)). These authors consider a variety of backward looking mechanisms for the formation of the expectations $p_{t}^{e}$ ranging across the traditional naive expectation $p_{t}^{e}=p_{t-1}$, learning expectations (e.g., learning by arithmetic mean $\left.p_{t}^{e}=\left(p_{t-1}+\cdots+p_{t-L}\right) / L\right)$ and adaptive learning expectation $p_{t}^{e}=$ $p_{t-1}^{e}+w\left(p_{t-1}-p_{t-1}^{e}\right)$ with $0 \leq w \leq 1$. Assuming bounded rationality of agents, Hommes (1998) even shows that such simple expectation schemes can be consistent with rational behaviour in the nonlinear cobweb model.

When producers are somewhat uncertain about the dynamics of the economic system in which they are to play out their roles and exhibit bounded rationality, they need to engage in some learning scheme to update their beliefs. Among various possible learning schemes, the properties of recursive learning processes under homogeneous expectations have been studied extensively (e.g., Bray (1982, 1983), Evans and Ramey (1992), Balasko and Royer (1996), Evans and Honkapohja (1994, 1995, 1999), Barucci $(2000,2001))$. In Bray $(1982,1983)$ and Evans and Honkapohja (1994, 1995), the agent's expectation is computed as the arithmetic average of all the past observations with full memory (the same weight is employed for each observation). In Balasko and Royer (1996), agents' expectations are updated by finite recursive least squares processes (using a moving average of the past $h$ prices) and it is found that an equilibrium which is stable under learning with finite memory $h$ is also stable for a finite memory $h^{\prime}$ with $h^{\prime}>h$. Their results are extended further in Chiarella and He $(2003 b)$ to more general finite recursive processes with nonnegative weights and it is found that the stability of equilibrium depends on the weighting vector and that complicated dynamics can be generated. In Barucci (2000, 2001), agents' expectations are 
computed as a weighted average of all the past observations with no-full memory. The weights of the average are described by a geometric process with a ratio smaller than 1 and therefore, the weights for older observations are smaller than the weights for recent observations. As pointed by Barucci (p.234, 2001), these features of a fading memory learning mechanism are "appealing because...the assumption of a constant weight for past observations is not fully plausible from a behavioral point of view. As a matter of fact, agents do not stop to learn as time goes on and they 'forget' remote observations". For a class of nonlinear deterministic forward-looking economic models under fading memory learning, Barucci shows that the decay rate of the memory of the learning process plays a stabilizing role-an increase of the memory decay rate enlarges the local stability parameter region of the perfect foresight stationary equilibria.

Brock and Hommes (1997) study heterogeneity in expectation formation by introducing the concept of adaptive rational equilibrium dynamics (ARED). They consider a cobweb model in which agents choose a predictor from a finite set of expectations functions of past information and update their beliefs over time according to a publicly available 'fitness' measure. They demonstrate the so called rational route to randomness. This framework has been extended further to the heterogeneous cobweb model by allowing more types of agents(e.g. Branch (2002) and Onozaki et al $(2000,2003)$ ) and various learning among heterogeneous agents (e.g. Chiarella and $\mathrm{He}(2003 a)$ ).

Nonlinearity can also come from considerations of risk and risk aversion, as discussed in Boussard and Gerard (1991), Burton (1993) and Boussard (1996). Boussard (1996) points out that with risk averse producers, the traditional linear cobweb model becomes nonlinear. By assuming that the actual price $p_{t}$ is uncertain so that $p_{t}^{e}$ has mean $\bar{p}_{t}$ and variance $\bar{v}_{t}$, Boussard (1996) shows that, under the simplest learning scheme $\bar{p}_{t}=\hat{p}$ and $\bar{v}_{t}=\left(p_{t-1}-\hat{p}\right)^{2}$ with constant $\hat{p}$, the nonlinear model may result in the market generating chaotic price series, and market failure, and therefore the source of risk is the risk itself (p.435, Boussard (1996)). Consequently, the study "casts a new light on expectations. Not only are expectations pertaining to mean values important for market outcomes. Those pertaining to variability can be just as crucial" (p.445, Boussard (1996)).

Apart from Boussard (1996), the focus in the expectations formation literature has been on schemes for the mean, with very little attention being paid to schemes for the variance. Chiarella and $\mathrm{He}$ (2000) extend Boussard's framework in a way that takes account of the risk aversion of producers and allows them to estimate both the mean and variance via an arithmetic learning process (ALP). They show that the resulting cobweb dynamics form a complicated nonlinear expectations feedback structure whose dimensionality depends upon the length of the window of past prices (the lag length) used to estimate the moments of the price distributions. It is found that an increase of the window length can enlarge the parameter region of the local stability of the steady state and, at the crossover from local stability to local instability, the dynamics exhibit resonance behavior which is indicative of quite complicated dynamical behavior, and even chaos (for the model with constant elasticity supply and demand functions).

Motivated largely by the above literature in heterogeneous expectations and learning, this paper aims to study the dynamics of the cobweb model with risk averse heterogeneous producers who adopt fading memory learning processes. We first extend the homogeneous model in Chiarella and $\mathrm{He}$ (2000) to incorporate heterogeneous agents. 
By allowing the heterogeneous producers to follow geometric decay (learning) processes (GDP, see Section 2 for definition), we then study the role of the memory decay rate on the price dynamics. It is found that, when the memory is infinite, an increase of the memory decay rate plays a stabilizing role on the local stability of the steady state price, as also found in Barucci $(2000,2001)$ when agents are homogeneous. However, the effect of the memory decay rate becomes less clear when the memory is finite. The heterogeneity has a double edged effect on the price dynamics in the sense that heterogeneous learning can stabilize an otherwise unstable dynamics in some cases and destablize an otherwise stable dynamics in other cases as well. It is shown that (quasi)periodic solutions and strange (or even chaotic) attractors can be created through Neimark-Hopf bifurcations when the memory is infinite and through flip bifurcations as well when the memories are finite. In addition, it is found that the source of risk is the risk itself, as pointed out in Boussard (1996), in the sense that the behaviour of producers in response to risk can generate market failure.

The paper is organised as follows. A general cobweb model with heterogeneous producers is established in Section 2. The heterogeneous geometric decay (learning) processes (GDP) is introduced, and the existence of steady-state (rational equilibrium) is also discussed. Section 3 considers the dynamics of the heterogeneous model with standard arithmetic learning process (ALP) as a special case of the GDP with finite memory. Then the dynamics of the model with heterogeneous GDP for both finite and infinite memories are analyzed in Sections 4 and 5, respectively. Section 6 concludes the paper.

\section{Cobweb Model with Heterogeneous Producers}

This section sets up a cobweb model in which there are $h$ producers who are heterogeneous in their risk attitudes and in their formulation of the expected mean and variance. In the case of linear supply and demand functions, the model may be written as

$$
\left\{\begin{array}{l}
\text { Supply: } \quad p_{i, t}^{e}=a_{i} q_{i, t}+b_{i}, \quad(i=1,2, \cdots, h) \\
\text { Demand: } \quad p_{t}=\alpha q_{t}+\mu \quad(\alpha<0)
\end{array}\right.
$$

where $q_{t}$ is the aggregate supply, $q_{i, t}$ and $p_{i, t}^{e}$ are the quantity supplied and price expected of producer $i$ at time $t$ based on the information set at $t-1$, and $p_{t}$ is the market price, and $a_{i}, b_{i}, \mu(>0)$ and $\alpha<0$ are constants.

From the perspective of their information set at $t-1$, the price $p_{t}$ is uncertain so the heterogeneous producers treat $p_{i, t}^{e}$ as a random variable drawn from a normal distribution whose mean and variance they are seeking to learn ${ }^{1}$.

2.1. Market Clearing Price and Heterogeneous Model. Let $\bar{p}_{i, t}$ and $\bar{v}_{i, t}$ be, respectively, the subjective mean and variance of expected price $p_{i, t}^{e}$ of producer $i$ formed at time $t$ based on the information set at $t-1$, and $q_{t}$ be quantity at time $t$. With constant absolute risk aversion $A_{i}$, the marginal revenue certainty equivalent of producer $i$ is

\footnotetext{
${ }^{1}$ It would of course be preferable (and more in keeping with models of asset price dynamics in continuous time finance) to treat $p_{i, t}^{e}$ as log-normally distributed. However this would then move us out of the mean-variance framework so we leave an analysis of this approach to future research.
} 
given by ${ }^{2}$

$$
\tilde{p}_{i, t}=\bar{p}_{i, t}-2 A_{i} \bar{v}_{i, t} q_{i, t} .
$$

Assume a linear marginal cost, as in (2.1), so that the supply equation, under marginal revenue certainty equivalence, becomes

$$
\tilde{p}_{i, t}=a_{i} q_{i, t}+b_{i} .
$$

Equating (2.2) and (2.3) determines the supply for producer $i$ as

$$
q_{i, t}=\frac{\bar{p}_{i, t}-b_{i}}{a_{i}+2 A_{i} \bar{v}_{i, t}} .
$$

Denote by $n_{i}$ the proportion of type $i$ producers $^{3}$, then the market clearing price is determined by ${ }^{4}$

$$
p_{t}=\mu+\alpha \sum_{i} n_{i} \frac{\bar{p}_{i, t}-b_{i}}{a_{i}+2 A_{i} \bar{v}_{i, t}} .
$$

In the rest of this paper, the simplest heterogeneous model with two types of producers is considered. Then the population of heterogeneous producers can be measured by a single parameter $w=n_{1}-n_{2}$, which is the proportional difference between the two types. Correspondingly, equation (2.5) can be rewritten as

$$
p_{t}=\mu+\frac{\alpha}{2}(1+w) \frac{\bar{p}_{1, t}-b_{1}}{a_{1}+2 A_{1} \bar{v}_{1, t}}+\frac{\alpha}{2}(1-w) \frac{\bar{p}_{2, t}-b_{2}}{a_{2}+2 A_{2} \bar{v}_{2, t}} .
$$

2.2. Heterogeneous Learning Processes. The heterogeneous model (2.6) is incomplete until producers' expectations are specified. In this paper, geometric decay processes (GDP) with either finite and infinite memory are assumed. More precisely, for type $i$ producers, the GDP with finite memory is defined by assuming that the conditional mean and variance of the price follows a geometric probability distribution with decay rate of $\delta_{i}$ over a window length of $L_{i}$, that is,

$$
\left\{\begin{aligned}
\bar{p}_{i, t} \equiv m_{i, t-1} & =B_{i} \sum_{j=1}^{L_{i}} \delta_{i}^{j-1} p_{t-j}, \\
\bar{v}_{i, t} \equiv v_{i, t-1} & =B_{i} \sum_{j=1}^{L_{i}} \delta_{i}^{j-1}\left[p_{t-j}-m_{i, t-1}\right]^{2},
\end{aligned}\right.
$$

where $B_{i}=1 /\left(1+\delta_{i}+\delta_{i}^{2}+\cdots+\delta_{i}^{L_{i}-1}\right), L_{i} \geq 1$ are integers, and $\delta_{i} \in[0,1]$ are constants for $i=1,2$. Two special cases of the GDP are of particular interest. When $\delta_{1}=\delta_{2}=0$, the expectation of the mean follows the naive expectation $\bar{p}_{i, t}=p_{t-1}$

\footnotetext{
${ }^{2}$ With constant absolute risk aversion $A_{i}$, we assume the certainty equivalent of the receipts $r=p q$ is $R\left(q_{t}\right)=\bar{p}_{i, t} q_{t}-A_{i} \bar{v}_{i, t} q_{t}^{2}$. Then maximisation of this function with respect to $q_{t}$ leads to the marginal revenue certainty equivalent $\tilde{p}_{t}=\frac{\partial R}{\partial q_{t}}=\bar{p}_{i, t}-2 A_{i} \bar{v}_{i, t} q_{t}$. We recall that this objective function is consistent with producers maximising the expected utility of receipts function $U_{i}(r)=-e^{-A_{i} r}$.

${ }^{3}$ In general, the proportion $n_{i}$ is a function of time $t$, that is, $n_{i, t}$, which can be measured by a certain fitness function and discrete choice probability, as in Brock and Hommes (1997). Because of the complexity of the analysis, we consider only the case with fixed proportions and leave the changing proportion case for future work.

${ }^{4}$ From (2.1) and (2.4), the aggregate supply is given by $q_{t}=\sum n_{i} q_{i, t}=\left(p_{t}-\mu\right) / \alpha$, from which (2.5) follows upon use of (2.4).
} 
and $\bar{v}_{i, t}=0$. When $\delta_{1}=\delta_{2}=1$, the GDP (2.7) is reduced to the standard arithmetic learning process $(A L P)$,

$$
\bar{p}_{i, t}=\frac{1}{L_{i}} \sum_{j=1}^{L_{i}} p_{t-j}, \quad \bar{v}_{i, t}=\frac{1}{L_{i}} \sum_{j=1}^{L_{i}}\left[\bar{p}_{i, t}-p_{t-j}\right]^{2} .
$$

As memory becomes infinite, that is, as $L_{i} \rightarrow \infty$, it is shown (see Appendix A) that, as a limiting process of GDP with finite memory, the GDP with infinite memory has the form

$$
\left\{\begin{aligned}
m_{i, t} & =\delta_{i} m_{i, t-1}+\left(1-\delta_{i}\right) p_{t}, \\
v_{i, t} & =\delta_{i} v_{i, t-1}+\delta_{i}\left(1-\delta_{i}\right)\left(p_{t}-m_{i, t-1}\right)^{2} .
\end{aligned}\right.
$$

2.3. Existence of the Unique Steady State Price. Denote by $p^{*}$ the state steady price of the GDP model with finite memory. It is found from (2.6) that $p^{*}$ satisfies

$$
p^{*}=\frac{\mu-\frac{\alpha}{2}\left[(1+w) \frac{b_{1}}{a_{1}}+(1-w) \frac{b_{2}}{a_{2}}\right]}{1-\frac{\alpha}{2}\left[(1+w) \frac{1}{a_{1}}+(1-w) \frac{1}{a_{2}}\right]} .
$$

For the GDP model with infinite memory, the state steady is given by $\left(p_{t}, m_{i, t}, v_{i, t}\right)=$ $\left(p^{*}, p^{*}, 0\right)$.

In the following sections, the dynamics of the heterogeneous model (2.6) are studied first when agents update their estimates of both the mean and variance by using the ALP (2.8). The analysis is then generalised to the GDP (2.7) with finite memory and (2.9) with infinite memory .

\section{Dynamics of the Heterogeneous Cobweb Model with AlP}

As a special case of the heterogeneous model with finite GDP, this section focuses on the case where producers have full finite memory about the history of prices, that is $\delta_{1}=\delta_{2}=1$. Correspondingly, the GDP is reduced to ALP, which has been studied in the recent literature (e.g. Balasko and Royer (1996) and Chiarella and He (2003b)). Without loss of generality, we assume $L_{1} \leq L_{2}$ and denote $L=\max \left\{L_{1}, L_{2}\right\}=$ $L_{2}$. Because of the dependence of the subjective mean $\bar{p}_{t}$ and variance $\bar{v}_{t}$ on price lagged $L$ periods, equation (2.6) is a difference equation of order $L$ (see system (B.2) in Appendix B).

The local stability of the unique steady state $p_{t}=p^{*}$ is determined by the eigenvalues of the corresponding characteristic equation (equation (B.3) in Appendix B), which is difficult to analyze in general. The following discussion first focuses on the case when $L_{1}=L_{2}=L$ and then some special cases when $L_{1} \neq L_{2}$ and $L_{1}, L_{2}=1,2,3,4$, in order to gain some insights into the effect of differing lag lengths on the regions of stability and the types of dynamic behaviour that may occur. As indicated from the following results, the local stability of the steady state depends on various parameters, including those from supply and demand functions $a_{1}, a_{2}, \alpha$, the proportional difference parameter $w$, and the window lengths $L_{1}$ and $L_{2}$ used by the heterogeneous producers. The discussion here focuses on two different aspects. On the one hand, for a fixed window length combination of $\left(L_{1}, L_{2}\right)$, we consider how the demand parameter $\alpha$ and the proportional difference $w$ of producers affect the local stability of the steady state and bifurcation. On the other hand, for a set of fixed parameters, we examine how these results on the local stability and bifurcation are affected by different combinations of the window lengths. It is found from the following discussion that both the 
local stability region and bifurcation boundary are geometrically easy to construct by using parameters $\beta_{1}$ and $\beta_{2}$, defined by

$$
\beta_{1}=-\frac{\alpha}{2 a_{1}}(1+w), \quad \beta_{2}=-\frac{\alpha}{2 a_{2}}(1-w),
$$

instead of $w$ and $\alpha$. However, the one-one relation (3.1) between $(w, \alpha)$ and $\left(\beta_{1}, \beta_{2}\right)$ makes it possible to transform the results between the different set of parameters and to preserve the geometric relation of the local stability regions between the two sets of parameter. ${ }^{5}$ In the following discussion, for the reason just stated, the results are formulated in terms of $\left(\beta_{1}, \beta_{2}\right)$, although some of the stability regions are also plotted using $(w, \alpha)$.

3.1. Case 1: $L_{1}=L_{2}=L$. When both types of producer use the same window length, that is $L_{1}=L_{2}=L$, using the Lemma in Chiarella and He (2003a), a relatively complete result on both the local stability region of the steady state and the types of bifurcation for general lag length $L$ can be stated as follows.

Proposition 3.1. For the nonlinear system (2.6), assume producers follow ALP and $L_{1}=L_{2}=L$. Then the steady state $p^{*}$ is locally asymptotically stable (LAS) if

$$
0 \leq-\frac{\alpha}{2}\left(\frac{1+w}{a_{1}}+\frac{1-w}{a_{2}}\right)<L, \quad \text { i.e., } \quad 0 \leq \beta_{1}+\beta_{2}<L .
$$

Furthermore, the boundary $\beta_{1}+\beta_{2}=L$ defines a $1:(L+1)$ resonance bifurcation. ${ }^{6}$

In terms of the effect of lag length $L$ on the local stability region of the steady state, an analysis on the stability boundary $\beta_{1}+\beta_{2}=L$ leads to the following Corollary (see Chiarella and $\mathrm{He}(2003 a)$ for more discussion).

Corollary 3.2. For the nonlinear system (2.6), assume producers follow $A L P$ and $L_{1}=$ $L_{2}=L$. Then, in terms of the parameters $\alpha$ and $w$, increasing $L$ can stabilise the otherwise unstable steady state.

To illustrate the periodicity of different resonance bifurcations, typical time series for $L=2,5$ and 10 are plotted in Figure 3.1. Similar plots (not reported here) are also found when $a_{1} \neq a_{2}$.

\footnotetext{
${ }^{5}$ Note that the determinant of the Jacobian of the transformation (3.1) does not change the sign, implying the preservation of the transformation.

${ }^{6}$ Resonance bifurcations occur when the eigenvalues lie on the unit circle. When $\beta_{1}+\beta_{2}=L$, the eigenvalues are given by $\lambda_{k}=e^{2 k \mu \pi i}$ with $\mu=1 /(L+1)$. Geometrically, the $L$ eigenvalues correspond to the $L+1$ unit roots distributed evenly on the unit circle, excluding $\lambda=1$. When $L=1$, a flip or period-doubling bifurcation occurs. When $L=2$, according to Kuznetsov (1995), the bifurcation is known as a 1:3 strong resonance, leading to two sets of period three cycles with one set stable and other set unstable (see Chiarella and He (2000) for more details). For $L \geq 2$, according to Sonis (2000), the bifurcation is given by $1: L+1$ periodic resonances. For $L_{1}=L_{2}=L=3,4$, instability of the steady state leads to 1:4 and 1:5 periodic resonance bifurcations, respectively, and similar dynamics to 1:3 resonance bifurcation are also found. Theoretical analysis for such types of bifurcation of higher dimensional discrete systems can be exceedingly complicated and not yet completely understood, (see Example 15.34 in Hale and Kocak (pp. 481-482, (1991)))
} 


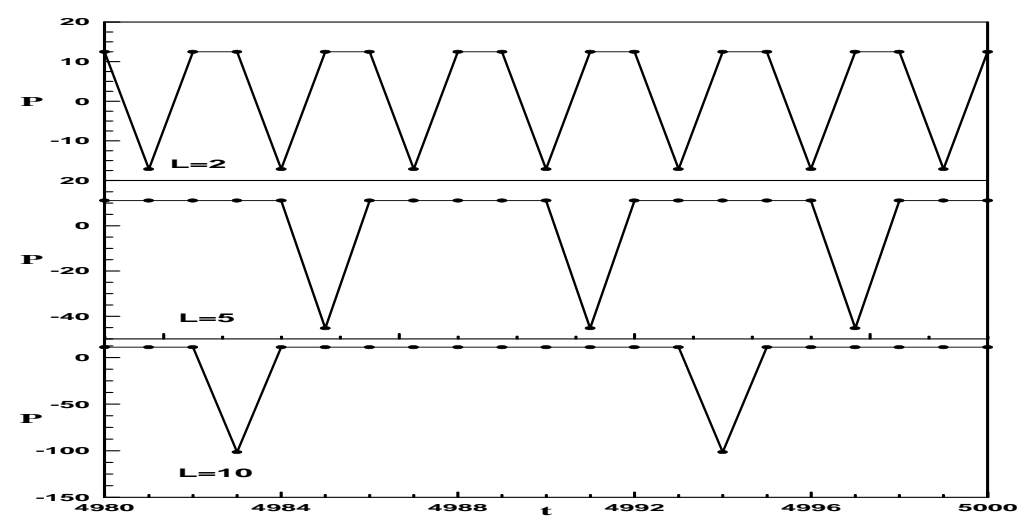

FIGURE 3.1. Time series plots of the nonlinear system (2.6) for $L=$ $2, \alpha=-2$ (the upper panel); $L=5, \alpha=-4.5$ (the middle panel); and (c) $L=10, \alpha=-9$ (the lower panel). Here $\beta=11, a_{1}=a_{2}=1, A=$ $0.005, w=0, b_{1}=b_{2}=0$ and $L_{1}=L_{2}=L=2$.

3.2. Case 2: $L_{1} \neq L_{2}$. For $L_{1}<L_{2}=L$, comparing with the case of $L_{1}=L_{2}$, the local stability regions of the steady state and bifurcation boundaries for different combination of lag lengths have less clear features and indeed become very complicated and difficult to analyse in general. A detailed analysis on the stability conditions and types of bifurcations for $L_{i}=1,2,3,4(i=1,2)$ can be found in Chiarella, He and Zhu (2003) and the stability results are illustrated in Figure 3.2. Denote by $D_{L_{1} L_{2}}$ the local stability region in terms of parameters $\left(\beta_{1}, \beta_{2}\right)$. In general, an increase of window length (either $L_{1}$ or $L_{2}$ ) enlarges the parameter region of the local stability of the steady state in general (e.g, $D_{11} \subset D_{13} \subset D_{14} \subset D_{24}, D_{22} \subset D_{24}$ ). However, this is not always true, such as $D_{12} \nsubseteq D_{13}, D_{12} \nsubseteq D_{22}, D_{34} \nsubseteq D_{44}$.
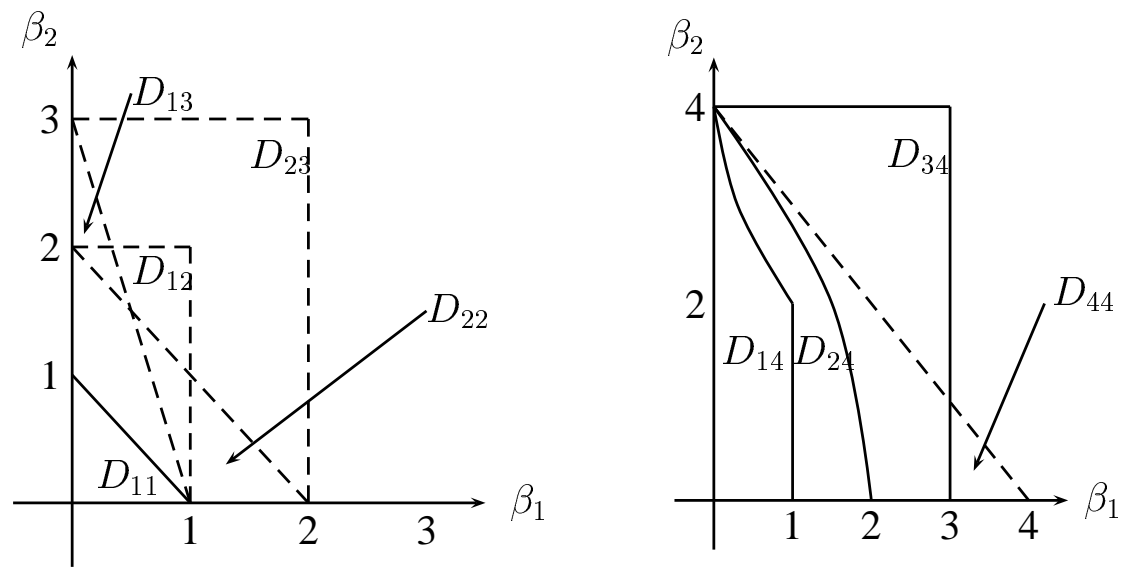

FIGURE 3.2. The local stability regions $D_{L_{1} L_{2}}$ of the steady state and bifurcation boundaries for various $\left(L_{1}, L_{2}\right)$ with $L_{1} \leq L_{2}=1,2,3$.

Types of bifurcation. For $\left(L_{1}, L_{2}\right)=(1,2),(1,4),(2,3)$ and $(3,4)$, the steady state becomes unstable through either a flip or Hopf bifurcation. However, for $\left(L_{1}, L_{2}\right)=$ $(1,3)$, the stability region is bounded by the flip bifurcation boundary only, while for $\left(L_{1}, L_{2}\right)=(2,4)$, the stability region is bounded by the Hopf bifurcation boundary only. The variety of types of bifurcation and complexity of the dynamics is 


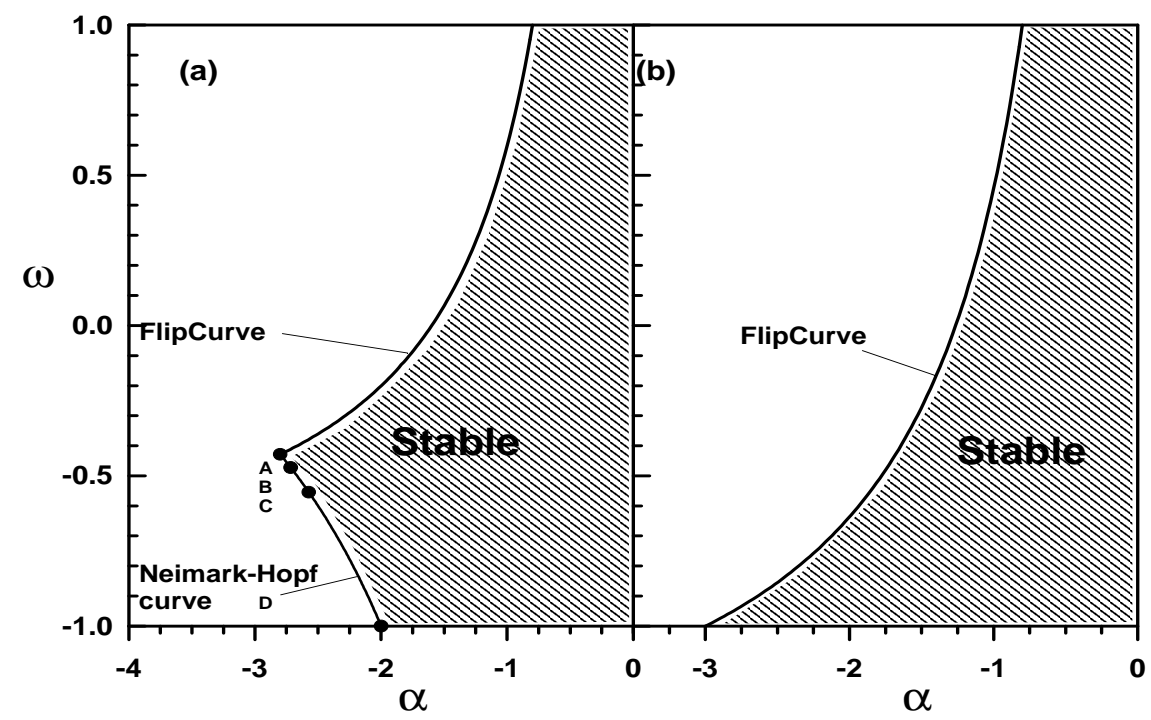

FIGURE 3.3. The local stability regions of the steady state of the nonlinear system (2.6) for (a) $\left(L_{1}, L_{2}\right)=(1,2)$ and (b) $\left(L_{1}, L_{2}\right)=$ $(1,3)$ in $(\alpha, w)$ plane with parameters $\beta=11, a_{1}=0.8, a_{2}=1, A=$ $0.005, b_{1}=b_{2}=0$.

\begin{tabular}{|c|c|c|c|}
\hline$(p, q)$ & $\rho$ & $\left(\beta_{1}, \beta_{2}\right)$ & $(w, \alpha)$ \\
\hline$(1,2)$ & -2 & $(1,2)$ & $(-0.43,-2.8)$ \\
$(1,3)$ & -1 & $(0,2)$ & $(-1,-2)$ \\
$(2,5),(3,5)$ & -1.618 & $(0.618,2)$ & $(-0.60357,-2.49)$ \\
\hline$\theta=\sqrt{2}$ & -1.7164 & $(0.7164,2)$ & $(-0.554517,-2.57)$ \\
\hline
\end{tabular}

TABLE 1. Parameter values for various resonance and quasiperiodic bifurcation along the Hopf bifurcation boundary for ALP with $\left(L_{1}, L_{2}\right)=(1,2)$ and $a_{1}=0.8, a_{2}=1$.

demonstrated through the case $\left(L_{1}, L_{2}\right)=(1,2)$ in the following discussion (for the case $\left(L_{1}, L_{2}\right)=(2,3)$, see Chiarella, He and Zhu (2003) for more details.). For $\left(L_{1}, L_{2}\right)=(1,2)$, the stability region $D_{12}=\left\{\left(\beta_{1}, \beta_{2}\right) ; 0 \leq \beta<1,0<\beta_{2}<2\right\}$, which can be transformed to the parameter space $(\alpha, w)$, indicated in Figure 3.3(a). Along the boundary $\beta_{1}=1, \beta_{2} \in[0,2]$, one of the eigenvalues is $\lambda=-1$, implying that a flip bifurcation occurs along this boundary. Along the other boundary $\beta_{2}=2, \beta_{1} \in[0,1]$, the two eigenvalues $\lambda_{1,2}=e^{ \pm 2 \pi \theta i}$, satisfying $\rho \equiv \lambda_{1}+\lambda_{2}=$ $2 \cos (2 \pi \theta)=-\left(\beta_{1}+\beta_{2} / 2\right), \lambda_{1} \lambda_{2}=\beta_{2} / 2=1$, and hence, the Neimark-Hopf bifurcation boundary is defined by $\beta_{1}=-1-\rho, \beta_{2}=2$. It follows from $\beta_{1} \in[0,1]$ that $\rho \in[-2,-1]$. The nature of the Neimark-Hopf bifurcation are determined by the value of $\theta$ and hence of $\rho$. For $\theta=p / q$, a rational fraction, the so-called $p: q$-periodic resonance occurs. If $\theta$ is an irrational number, then one obtains quasi-periodic orbits. Table 1 sets up some of the corresponding parameter values of $(w, \alpha)$ which give different types of resonance orbits (with $(p, q)=(1,2),(1,3),(2,5),(3,5),(1,5),(4,5))$, and one quasi-periodic orbit (with $\theta=\sqrt{2}$ ).

The above local bifurcation analysis and the variety of types of bifurcation along the Neimark-Hopf boundary are demonstrated by our numerical simulations of the 

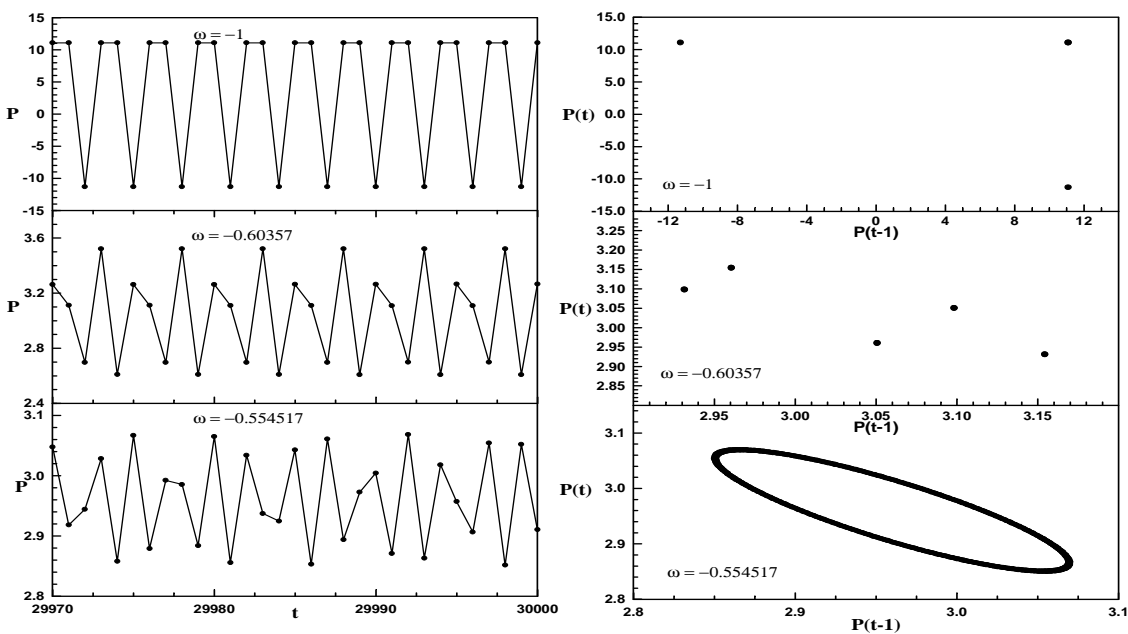

FIGURE 3.4. The time series (the left panel) and phase plots (the right panel) of periodic resonances of the nonlinear system (2.6) with $(p, q)=(1,3)$ and $(2,5)$, and quasi-periodic resonance with $\theta=\sqrt{2}$ for $L_{1}=1, L_{2}=2$ and $\beta=11, a_{1}=0.8, a_{2}=1, A=0.005, b_{1}=b_{2}=0$.

nonlinear cobweb system (2.6) when the parameter values are selected as indicated by Table 1. Points $D, B$ and $C$ in Figure 3.3(a) correspond to $1: 3$ and $2: 5$ resonances, and quasi-periodic closed orbit, respectively. For initial values near the steady state, the corresponding time series converge to the three time series plotted in the left panel in Figure 3.4. Corresponding to point $D$ and $B,(p, q)=(1,3)$ and $(2,5)$ or $(3,5)$, respectively, and the periodicity of the cycles of the time series are clearly identified by the time series (on the left panel) and phase plot (on the right panel) in Figure 3.4. In fact, for the point $B$, the phase plot indicates clearly two sets of period 5 cycles. For the point $C, \theta=\sqrt{2}$, solutions with initial values near the steady state converge to the quasi-periodic time series, on the bottom left panel. The quasi-periodicity of the time series is identified by the closed orbit of the phase plot, on the bottom right panel in Figure 3.4.

For fixed $\alpha=-2.494$ and $\left(L_{1}, L_{2}\right)=(1,2)$ and $(1,3)$, bifurcation diagrams with respect to the proportional population difference parameter $w$ are plotted in Figure 3.5. For $\left(L_{1}, L_{2}\right)=(1,3)$, the local stability region of the steady state of the nonlinear system (2.6) is shown in Figure 3.3(b). In this case, as $w$ increases, instability of the steady state leads to a flip type of bifurcation for a wide range of the parameter $w$, as indicated in the upper panel of Figure 3.5. However, for $\left(L_{1}, L_{2}\right)=(1,2)$, as $w$ decreases (from $w=-0.5$ ), instability of the steady state leads to more complicated and richer dynamics, indicated by the bifurcation diagram over the range of $w \in(-1,-0.6)$ in the lower panel of Figure 3.5.

In summary, for the nonlinear cobweb model with ALP, an increase of lag lengths plays a stabilizing role and the steady-state can become unstable through Hopf bifurcations only when both lags are the same. However when both lags are different, the stabilizing role becomes less clear and more complicated with either flip and Hopf bifurcation possibly occurring. Thus we may conclude that heterogeneity can stabilize an otherwise unstable dynamics in some cases and destabilize an otherwise stable dynamics in other cases. 


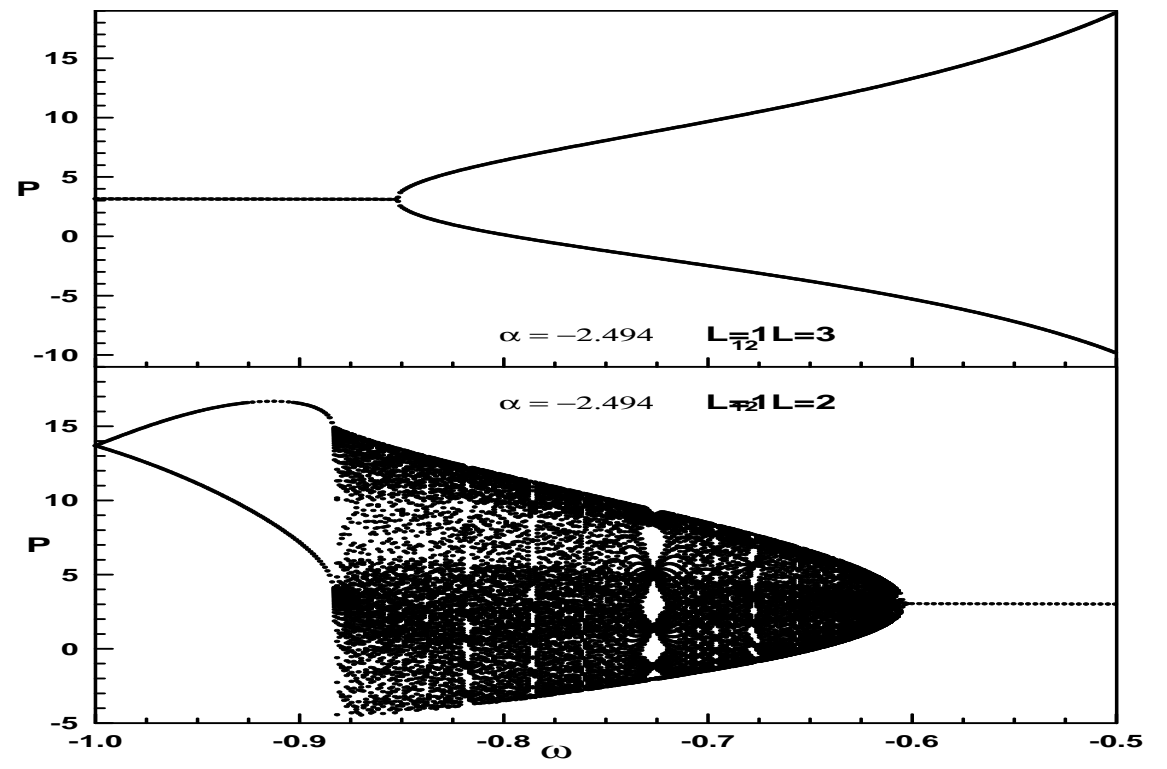

FIGURE 3.5. Bifurcation diagrams for fixed $\alpha=-2.494$ and (a) $\left(L_{1}, L_{2}\right)=(1,3),(\mathrm{b})\left(L_{1}, L_{2}\right)=(1,2)$, where $\beta=11, a_{1}=0.8, a_{2}=$ $1, A=0.005, b_{1}=b_{2}=0$.

\section{Dynamics of the Heterogeneous Model with Finite Memory GDP}

This section focuses on the dynamics of the nonlinear cobweb model (2.6) when producers follow the GDP with finite memory and different window lengths $L_{i}$. In the following discussion, we consider the case $L_{1}=L_{2}=L$ first and then the case $L_{1} \neq$ $L_{2}$. Because of the advantage of being able to easily display the results graphically, the analysis is again formulated in terms of $\left(\beta_{1}, \beta_{2}\right)$.

4.1. Case 1: $L_{1}=L_{2}=L$. Consider first the case when both types of producer use the same window length, that is $L_{1}=L_{2}=L$, but different decay rates $\left(\delta_{1}, \delta_{2}\right)$.

4.1.1. Local Stability and Bifurcation Analysis. The simplest case of $L=1$ can be treated as special case of GDP when the decay rate $\delta_{i}=0$, that is, agents use the traditional naive expectation, taking the latest price as their expected price for the next period. In this case, the condition for local stability is $0 \leq \beta_{1}+\beta_{2}<1$ (see Proposition 3.1) the steady state becomes unstable through a flip bifurcation, leading to a two-period cycle of two prices, one is above and one is below the steady state price.

In the case $L=2$, the following result can be stated.

Proposition 4.1. For $L_{1}=L_{2}=2$, the local stability region $D_{22}\left(\beta_{1}, \beta_{2}\right)$ of the state steady is defined by $D_{22}=\left\{\left(\beta_{1}, \beta_{2}\right): \Delta_{1}<1, \Delta_{2}<1\right\}$, where

$$
\Delta_{1}=\frac{\delta_{1}}{1+\delta_{1}} \beta_{1}+\frac{\delta_{2}}{1+\delta_{2}} \beta_{2}, \quad \Delta_{2}=\frac{1-\delta_{1}}{1+\delta_{1}} \beta_{1}+\frac{1-\delta_{2}}{1+\delta_{2}} \beta_{2} .
$$

Furthermore, a flip and Neimark-Hopf bifurcation occurs along the boundary $\Delta_{2}=1$ and $\Delta_{1}=1$, respectively.

Proof. See Appendix C.1. 
Comparing with the case of $L=1$, the parameter (in terms of $\left(\beta_{1}, \beta_{2}\right)$ ) region on the local stability of the steady state is enlarged as $L$ increases from $L=1$ to $L=2$. Hence agents can learn the steady state price over a wide region of parameters as they follow the GDP with $L=2$. However, as we shall see, these learning process, can generate far more complicated dynamics when the steady state price becomes unstable. To understand the effect of parameters $\beta_{i}$ and $\delta_{i}(i=1,2)$ on the stability of the state steady and types of bifurcation, we now undertake a more detailed analysis by considering various cases in terms of the parameters $\left(\delta_{1}, \delta_{2}\right)$.

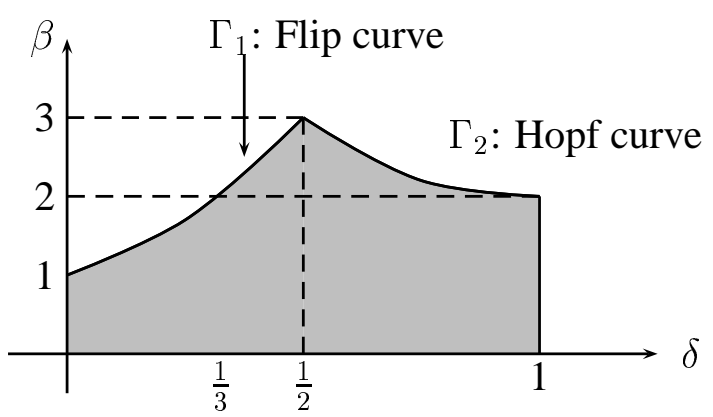

FIGURE 4.1. Stability region and bifurcation boundaries for $L_{1}=$ $L_{2}=2, \delta_{1}=\delta_{2}=\delta$ and $\beta=\beta_{1}+\beta_{2}$.

The case $\delta_{1}=\delta_{2}=\delta$ : In this case, from Proposition 4.1, the stability region of the state steady can be characterized by two parameters $\beta$ and $\delta$ with $D_{22}=\left\{\left(\beta_{1}, \beta_{2}\right)\right.$ : $\left.0 \leq \beta \equiv \beta_{1}+\beta_{2}<\bar{\beta}\right\}$ and $\bar{\beta}=\frac{1+\delta}{1-\delta}$ for $\delta \leq \frac{1}{2}$ and $\bar{\beta}=\frac{1+\delta}{\delta}$ for $\delta>\frac{1}{2}$. In this case, a flip bifurcation occurs along the boundary

$$
\Gamma_{1}: \beta=(1+\delta) /(1-\delta), \quad \delta \in[0,1 / 2],
$$

and a Neimark-Hopf bifurcation occurs along the boundary

$$
\Gamma_{2}: \beta=(1+\delta) / \delta, \quad \delta \in(1 / 2,1], \quad \rho=-1 / \delta \in(-2,-1] .
$$

The stability region $D_{22}$ is plotted ${ }^{7}$ in Figure 4.1, indicating various effects on stability of the decay rate $\delta$ :

(i) for $\delta \in\left[0, \frac{1}{2}\right]$, the stability region $D_{22}$ in terms of the parameter $\beta$ is enlarged as $\delta$ increases, and the steady state price becomes unstable through a flip bifurcation (implying a two-period cycle).

(ii) for $\delta \in\left[\frac{1}{2}, 1\right]$, the stability region $D_{22}$ in terms of the parameter $\beta$ is enlarged as $\delta$ decreases, and the steady state price become unstable through a NeimarkHopf bifurcation, which in turn generates either periodic cycles or aperiodic orbits.

(iii) for $\delta=0$, we have the smallest parameter $\beta$ region for local stability: $0 \leq$ $\beta<1$; while for $\delta=1 / 2$, we have the largest parameter $\beta$ region for the local stability: $0 \leq \beta<3$.

${ }^{7}$ Note that functions $f(x)=\frac{1+x}{1-x}, g(x)=\frac{1+x}{x}$ satisfy $f^{\prime}>0, f^{\prime \prime}>0, g^{\prime}<0, g^{\prime \prime}>0$. 


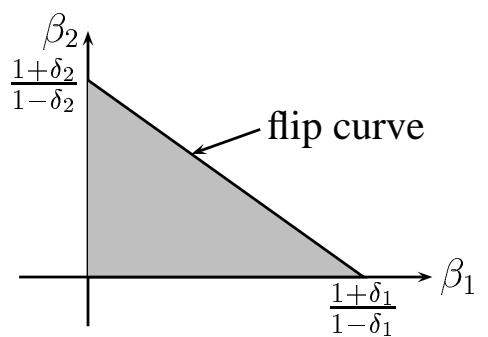

(a) $\delta_{1} \neq \delta_{2}, 0<\delta_{1}, \delta_{2}<\frac{1}{2}$

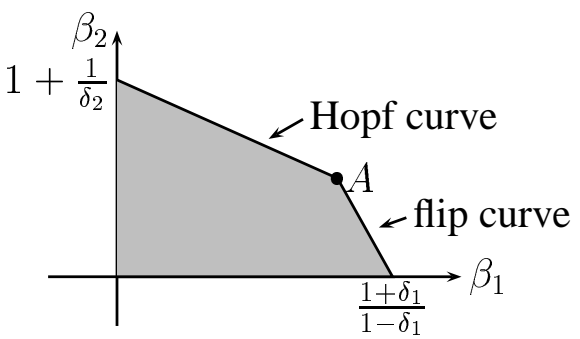

(c) $\delta_{1} \neq \delta_{2}, \delta_{1}<\frac{1}{2}, \delta_{2}>\frac{1}{2}$

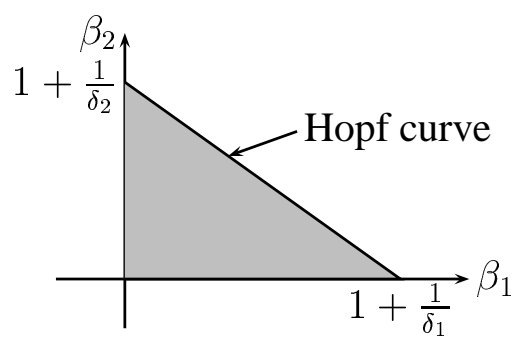

(b) $\delta_{1} \neq \delta_{2}, \frac{1}{2}<\delta_{1}, \delta_{2}<1$

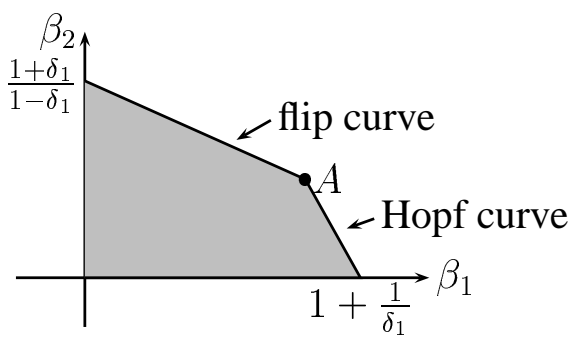

(d) $\delta_{1} \neq \delta_{2}, \delta_{1}>\frac{1}{2}, \delta_{2}<\frac{1}{2}$

FIGURE 4.2. Stability region and bifurcation boundaries for (a) $0 \leq$ $\delta_{1}, \delta_{2} \leq 1 / 2$; (b) $1 / 2<\delta_{1}, \delta_{2} \leq 1$; (c) $0 \leq \delta_{1} \leq 1 / 2<\delta_{2} \leq 1$; and (d) $0 \leq \delta_{2} \leq 1 / 2<\delta_{2} \leq 1$, where $A:\left(\beta_{1}, \beta_{2}\right)=\left(\left(1-2 \delta_{2}\right)\left(1+\delta_{1}\right) /\left(\delta_{1}-\right.\right.$ $\left.\delta_{2}\right),\left(1-2 \delta_{1}\right)\left(1+\delta_{2}\right) /\left(\delta_{2}-\delta_{1}\right)$.

The case $0 \leq \delta_{1}, \delta_{2}<1 / 2$ and $\delta_{1} \neq \delta_{2}$. In this case, it follows from Proposition 4.1 that the steady state becomes unstable through a flip bifurcation only, as indicated in Figure 4.2(a). Furthermore, as either $\delta_{1}$ or $\delta_{2}$ increases, the local stability region $D_{22}$ of the state steady with respect to parameters $\left(\beta_{1}, \beta_{2}\right)$ is enlarged, as indicated in Figure 4.3(b) where the stability region in $\left(\delta_{1}, \beta_{1}, \beta_{2}\right)$ is plotted for fixed $\delta_{2}=1 / 3$.

The case $\delta_{1}, \delta_{2}>1 / 2$ and $\delta_{1} \neq \delta_{2}$. In this case, it follows from Proposition 4.1 that the steady state becomes unstable through a Neimark-Hopf bifurcation, as indicated in Figures 4.2(b) and 4.3(a) where the stability region in $\left(\delta_{1}, \beta_{1}, \beta_{2}\right)$ is plotted for fixed $\delta_{2}=2 / 3$. Along the bifurcation boundary, the nature of the bifurcation is characterised by $\theta$ which satisfies (see Appendix C for the details) $\rho \equiv 2 \cos (2 \pi \theta) \in$ $\left(-1 / \min \left(\delta_{1}, \delta_{2}\right),-1 / \max \left(\delta_{1}, \delta_{2}\right)\right)$.

The case either $0<\delta_{1}<1 / 2, \delta_{2}>1 / 2$ or $0<\delta_{2}<1 / 2, \delta_{1}>1 / 2$. In this case, the stability region is bounded by two bifurcation boundaries, the flip bifurcation boundary $\Delta_{2}=1$ and the Neimark-Hopf bifurcation boundary $\Delta_{1}=1$, as indicated in Figures 4.2(c), (d) and 4.3(a)-(b). Also, the local stability region in $\left(\beta_{1}, \beta_{2}\right)$ is enlarged as either $\delta_{1}$ increases and $\delta_{2}$ decreases or $\delta_{2}$ increases and $\delta_{1}$ decreases.

Proposition 4.1 seems to indicate that as $L$ increases from 1 to 2 , on the one hand, the stability region is enlarged and, on the other hand, instability leads to a more complicated price dynamics through either flip or Hopf bifurcation. One may expect a similar effect to occur as $L$ increases from 2 to 3 . However, the following Proposition 4.2 indicates that this may not be the case. 

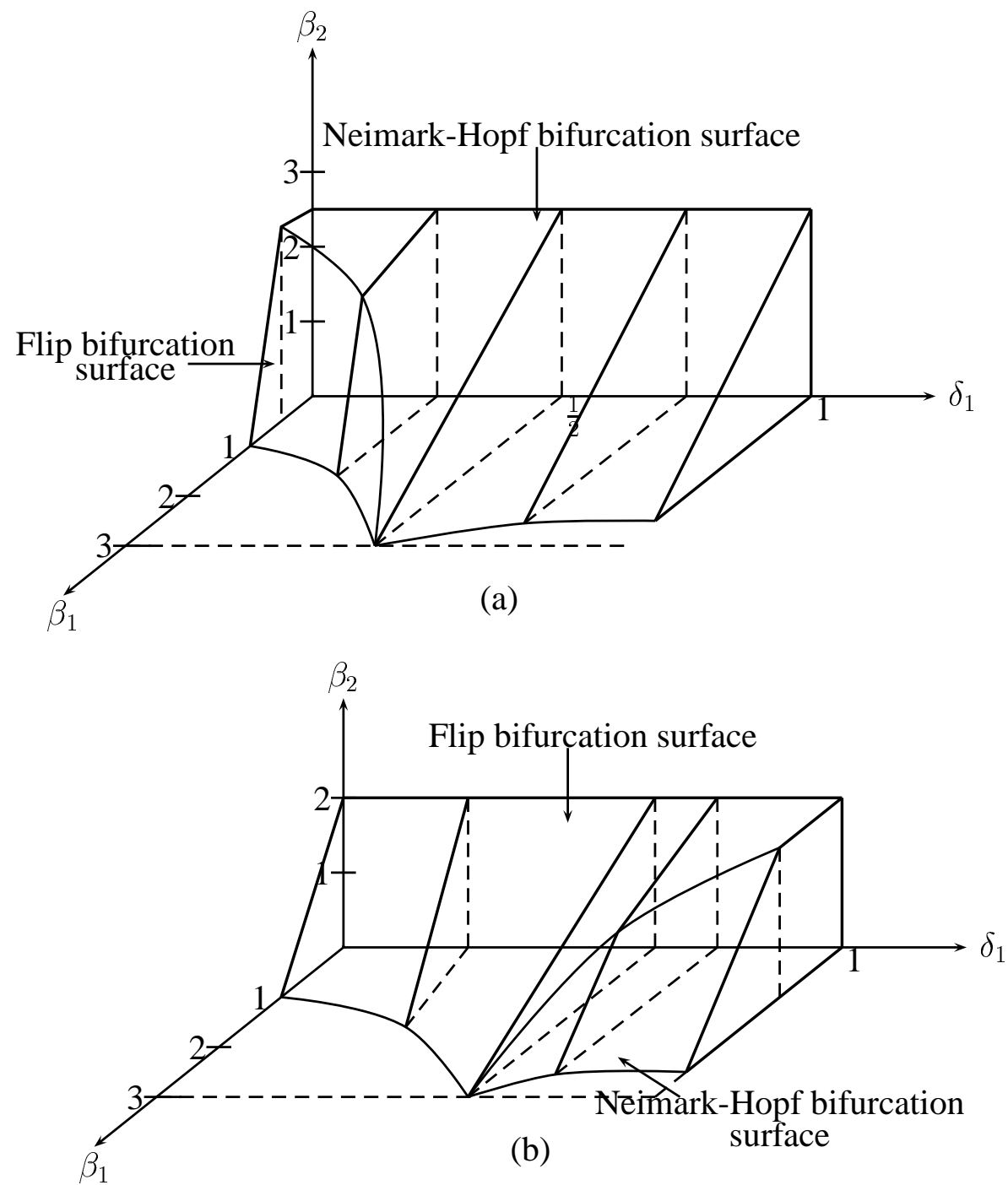

FIGURE 4.3. Stability region and bifurcation boundary surfaces for (a) $\delta_{2}=2 / 3$, and (b) $\delta_{2}=1 / 3$.

Proposition 4.2. For $L_{1}=L_{2}=3$, the local stability region $D_{33}\left(\beta_{1}, \beta_{2}\right)$ of the state steady is defined by $D_{33}=\left\{\left(\beta_{1}, \beta_{2}\right): \Delta_{3}<1\right\}$, where

$$
\Delta_{3}=\frac{1-\delta_{1}+\delta_{1}^{2}}{1+\delta_{1}+\delta_{1}^{2}} \beta_{1}+\frac{1-\delta_{2}+\delta_{2}^{2}}{1+\delta_{2}+\delta_{2}^{2}} \beta_{2} .
$$

Furthermore, a flip bifurcation boundary occurs when $\Delta_{3}=1$.

Proof. See Appendix C.1.

It is interesting to see that, similar to the case of $L=1$, but different from the case of $L=2$, the steady state becomes unstable only through a flip bifurcation when $L=3$. Moreover, the parameter region for local stability is enlarged as the decay rates $\delta_{i}$ increase. The stability regions are plotted in Figure 4.4(a) for $\delta_{1}=\delta_{2}=\delta, \beta=\beta_{1}+\beta_{2}$ and Figure 4.4(b) for $\delta_{1} \neq \delta_{2}$ and fixed $\delta_{2}=1 / 2$. 


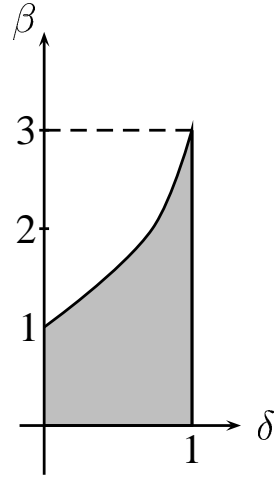

(a)

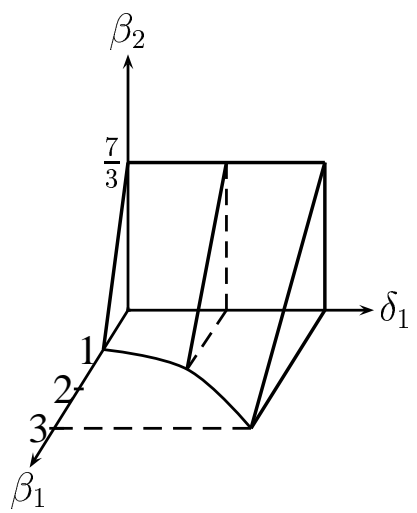

(b)

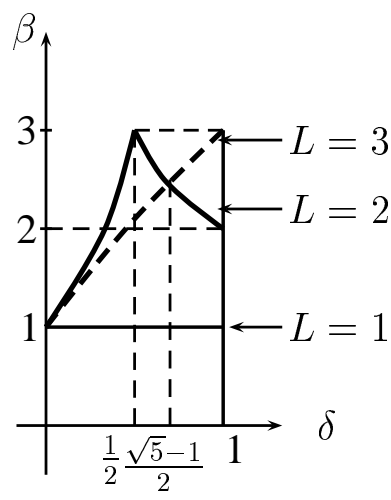

(c)

FIGURE 4.4. Stability region and bifurcation boundary (or surfaces) for $L=3$ and (a) $\delta_{1}=\delta_{2}=\delta, \beta=\beta_{1}+\beta_{2}$; (b) $\delta_{2}=1 / 2, \delta_{1} \in[0,1]$. (c) Comparison of the stability regions for $L=1,2,3$.

A general comparison among $L=1,2$ and 3 may not be easy for various $\delta_{1}$ and $\delta_{2}$. However, such comparison when $\delta_{1}=\delta_{2}=\delta$ can lead to some insight regarding the role of the decay rate on the price dynamics. In this case, the stability regions for $L=1,2$ and 3 are plotted in Figure 4.4(c) . One can see that: (i) for $\delta \in[0,1 / 2]$, the parameter $\beta$ region for the local stability of the steady state is enlarged as $\delta$ increases, $L=2$ leads to the largest stability region, and the steady state becomes unstable through a flip bifurcation; (ii) for $\delta \in(1 / 2,1], L=2$ gives a larger stability region for $\delta \leq(\sqrt{5}-1) / 2$, while $L=3$ gives a larger stability region for $\delta>(\sqrt{5}-1) / 2$. In addition, the steady state becomes unstable through a Neimark-Hopf bifurcation for $L=2$, but a flip bifurcation for $L=3$.

4.1.2. Dynamics of the Nonlinear System-Numerical Analysis. To illustrate the effect of the memory decay parameter on the dynamics, a bifurcation diagram with respect to the parameter $\delta_{2}$ is plotted in Figure 4.5 with parameters $L_{1}=L_{2}=2, \alpha=$ $-2.5, w=-0.6, \delta_{1}=0.15, a_{1}=0.8, a_{2}=1, A_{1}=A_{2}=0.005, \mu=11, b_{1}=b_{2}=0$. In particular, for $\delta_{2}=0.2$ and 0.88 , the phase plots and the corresponding time series are illustrated in Figure 4.6. For $\delta_{2}=0.2$, the prices converge to a two-period cycle, characterized by the flip bifurcation, while for $\delta_{2}=0.88$, the prices converge to a closed orbit in the phase plot, which is characterized by the Neimark-Hopf bifurcation.

It is interesting to see that the local stability condition and bifurcation in Propositions 4.1-4.2 are independent of the risk aversion coefficients $A_{i}$ of the heterogeneous agents. This is because these coefficients are associated with the variance, a higher order term of the linearised system of the nonlinear system at the steady state. In the above simulations in Figures 4.5 and 4.6, both the risk aversion coefficients are small, and hence the risk aversion and variance have no significant influence on the price dynamics induced from local stability analysis. When agents become more risk averse and more closely balance both mean and variance, one would expect the price dynamics to be stabilized in the sense that irregular price patterns, such as quasi-periodic cycles, with higher variability may become regular, such as cycles, with lower variability. This can be verified (not reported here) for the case corresponding to the right panel in 


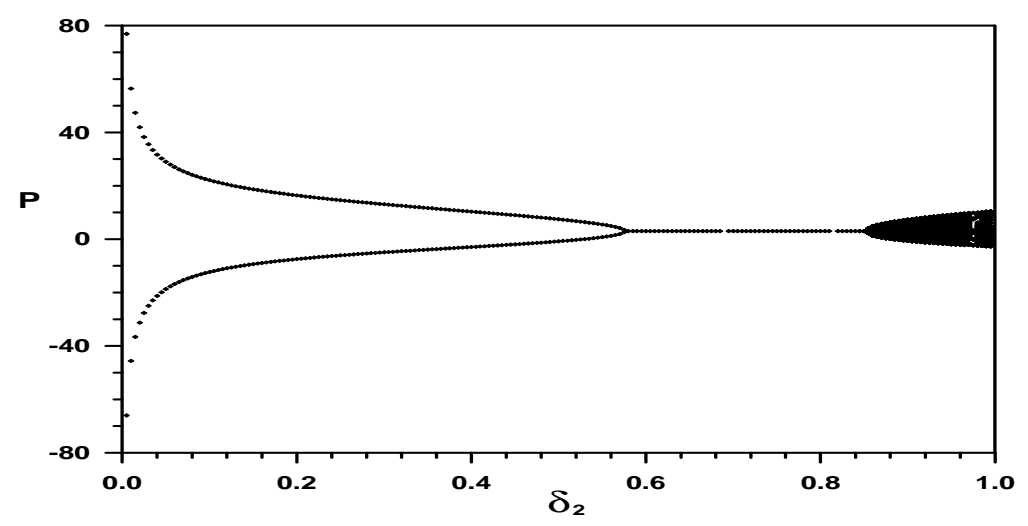

FIGURE 4.5. Bifurcation diagrams of the nonlinear system for $\delta_{2}$ with parameters $\alpha=-2.5, w=-0.6, \delta_{1}=0.15, a_{1}=0.8, a_{2}=1, A_{1}=$ $A_{2}=0.005, \beta=11, b_{1}=b_{2}=0, L_{1}=L_{2}=2$.

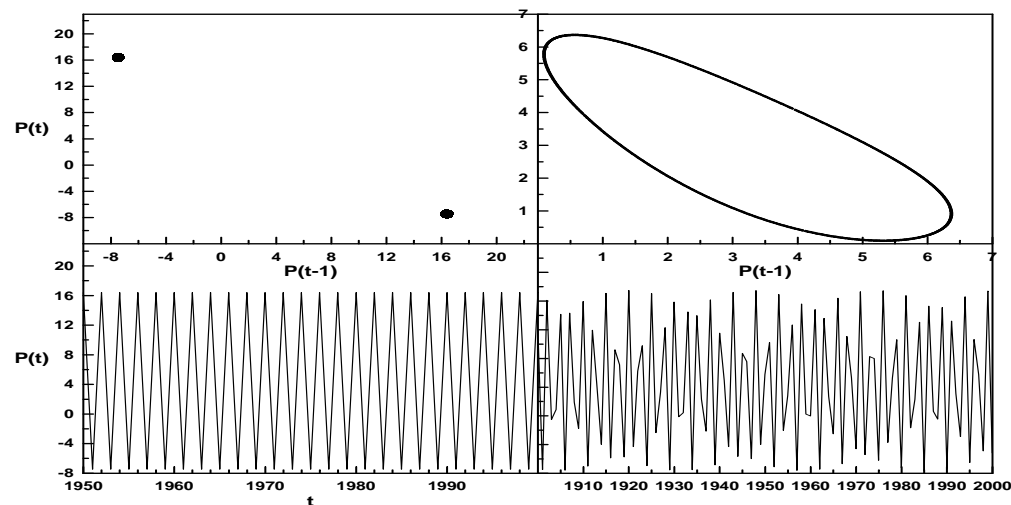

(a)

(b)

FIGURE 4.6. Phase plot and time series of the nonlinear system for (a) $\delta_{2}=0.2$ and (b) $\delta_{2}=0.88$ with parameters $\alpha=-2.5, w=-0.6, \delta_{1}=$ $0.15, a_{1}=0.8, a_{2}=1, A_{1}=A_{2}=0.005, \mu=11, b_{1}=b_{2}=0, L_{1}=$ $L_{2}=2$.

Figure 4.6, in which the steady state price becomes unstable through a Neimark-Hopf bifurcation and prices converge to the aperiodic pattern characterized by the closed orbit on the phase plot for small risk aversion coefficients $A_{1}=A_{2}=0.005$. As either $A_{1}$ or $A_{2}$ increases, the closed orbit becomes smaller (say for $A_{1}=A_{2}=A=0.01$ ). However, as $A_{i}$ increases further (say $A=0.05$ ) prices converge to, either aperiodic cycles (characterised by closed orbits for the phase plots) with lower variability for initial values near the steady state price, or 3-period cycles with higher variability for initial values not near the steady state price. Similar price dynamics are also observed when $\delta_{1}, \delta_{2}>1 / 2$. This suggests that, when the steady state price becomes unstable through a Neimark-Hopf bifurcation, an increase in the risk aversion can stabilise 
otherwise unstable price patterns initially and even leads to simple price dynamics. However, this is not necessarily true when the steady state price becomes unstable through a flip bifurcation.

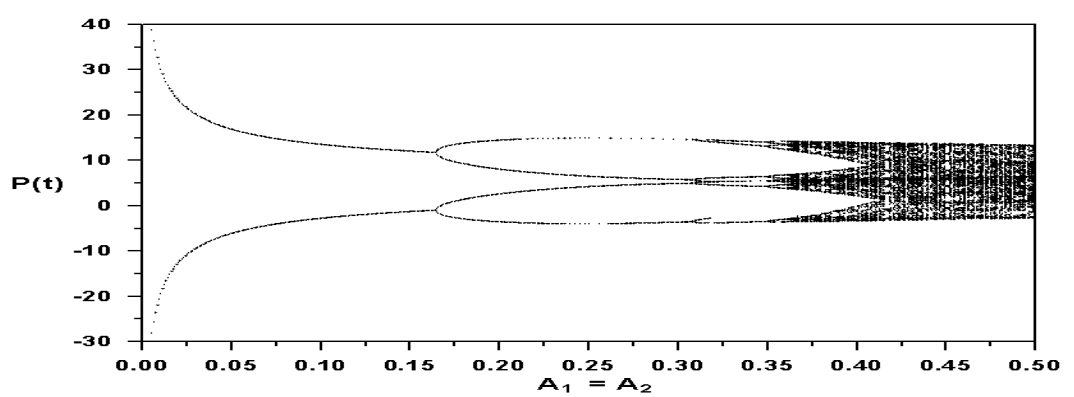

FIgURE 4.7. Bifurcation diagram in parameter $A=A_{1}=A_{2}$ with parameters $\delta_{1}=0.15, \delta_{2}=0.02, a_{1}=0.8<a_{2}=1, w=-0.6, \alpha=$ $-2.5, \beta=11, b_{1}=b_{2}=0, L_{1}=L_{2}=2$.

For the set of parameters: $\delta_{1}=0.15, \delta_{2}=0.02, \alpha=-2.5, \beta=11, b_{1}=b_{2}=$ $0, w=-0.6, a_{1}=0.8, a_{2}=1$, local stability analysis implies that the steady state price becomes unstable through a flip bifurcation when $\delta_{2}$ is small. This can be verified for $A_{i}$ small (say $A_{i}=0.005$ or 0.05 ), as indicated by the bifurcation diagram in parameter $A=A_{1}=A_{2}$ in Figure 4.7. As $A$ increases, the prices converge to period4 cycle for $A=0.2$, period- 8 cycle for $A=0.35$, period- 16 cycle for $A=0.36$, and a strange attractor for $A_{i}=0.5$. This strange attractor and the corresponding chaotic time series generated through such a flip bifurcation for $A_{i}=0.5$ are plotted in Figure 4.8 .

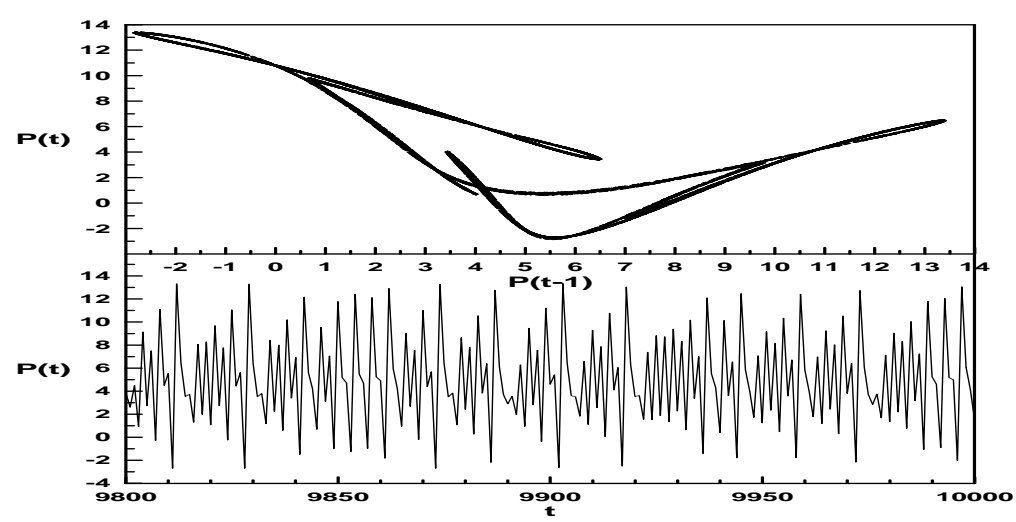

FIGURE 4.8. Phase plot and time series of the nonlinear system for $\alpha=-2.5, w=-0.6, \delta_{1}=0.15, \delta_{2}=0.02, a_{1}=0.8, a_{2}=1, A_{1}=$ $A_{2}=0.5, \beta=11, b_{1}=b_{2}=0, L_{1}=L_{2}=2$.

Based on this analysis, one can see that, risk aversion has a different effect on the price dynamics depending upon the type of bifurcation (flip or Hopf) through which 
the steady state price become unstable. When the steady state price becomes unstable through a Hopf bifurcation, as agents become more risk averse, the price dynamics become less complicated and the variability of the prices is reduced. However, when the steady state price becomes unstable through a flip bifurcation, as agents become more risk averse, the price dynamics become more complicated, although the variability of prices is reduced. It is in this sense that, as claimed by Boussard (1996), the source of the risk is the risk itself. Market price fluctuation and market failure can be generated when agents become more risk averse. This result is unexpected and interesting, and it underlines the connection between price dynamics generated by agents' risk and types of bifurcation.

4.2. Case 2: $L_{1} \neq L_{2}$. Consider now the case when both types of producer use the different window length $L_{1} \neq L_{2}$ and decay rates $\left(\delta_{1}, \delta_{2}\right)$.

4.2.1. Local Stability and Bifurcation Analysis. When $\delta_{1}=0$, the GDP with $\left(L_{1}, L_{2}\right)=$ $(2,2)$ and $(3,3)$ are reduced to the GDP with $\left(L_{1}, L_{2}\right)=(1,2)$ and $(1,3)$, respectively, and the stability region and the bifurcation boundaries in $\left(\delta_{2}, \beta_{1}, \beta_{2}\right)$ parameters space are plotted in Figure 4.9. For $\left(L_{1}, L_{2}\right)=(1,2)$, the stability region is bounded by a flip bifurcation surface for $\delta_{2} \leq 1 / 2$ and both flip and Neimark-Hopf bifurcation surfaces for $\delta_{2}>1 / 2$. For $\left(L_{1}, L_{2}\right)=(1,3)$, the stability region is bounded by a flip bifurcation surface only.

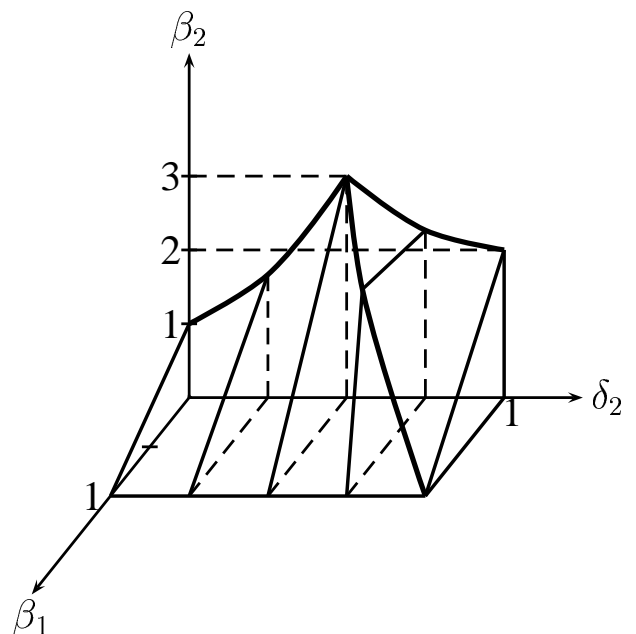

(a)

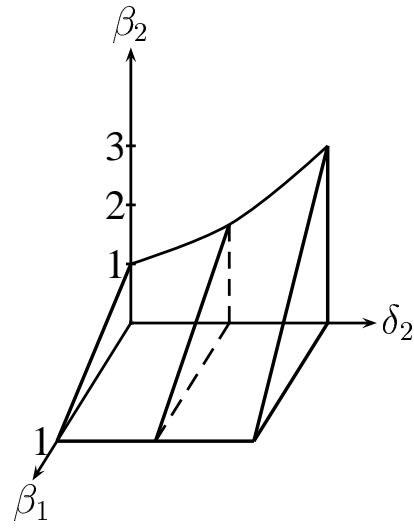

(b)

FIGURE 4.9. Stability region and bifurcation boundaries for (a) $\left(L_{1}, L_{2}\right)=(1,2)$, and (b) $\left(L_{1}, L_{2}\right)=(1,3)$.

For $\left(L_{1}, L_{2}\right)=(2,3)$, the following result can be obtained.

Proposition 4.3. For $L_{1}=2, L_{2}=3$, the stability region $D_{23}\left(\beta_{1}, \beta_{2}\right)$ of the steady state is defined by $D_{23}=\left\{\left(\beta_{1}, \beta_{2}\right): \Delta_{7}<1\right\}$ for $\delta_{1} \in[0,1 / 2]$ and $D_{23}=\left\{\left(\beta_{1}, \beta_{2}\right)\right.$ : 
$\left.\Delta_{7}<1, \Delta_{8}<1\right\}$ for $\delta_{1} \in(1 / 2,1]$, where

$$
\begin{aligned}
& \Delta_{7}=\frac{1-\delta_{1}}{1+\delta_{1}} \beta_{1}+\frac{1-\delta_{2}+\delta_{2}^{2}}{1+\delta_{2}+\delta_{2}^{2}} \beta_{2} \\
& \Delta_{8}=\frac{\delta_{1}}{1+\delta_{1}} \beta_{1}+\frac{\delta_{2}}{1+\delta_{2}+\delta_{2}^{2}} \beta_{2}-\frac{\delta_{2} \beta_{2}}{1+\delta_{2}+\delta_{2}^{2}}\left(\frac{\beta_{1}}{1+\delta_{1}}+\frac{\left(1-\delta_{2}^{2}\right) \beta_{2}}{1+\delta_{2}+\delta_{2}^{2}}\right) .
\end{aligned}
$$

Furthermore, for $\delta_{1} \in[0,1 / 2]$, a flip bifurcation occurs along the boundary $\Delta_{7}=$ 1; while for $\delta_{1} \in(1 / 2,1]$, both flip and Neimark-Hopf bifurcations occur along the boundary $\Delta_{7}=1$ and $\Delta_{8}=1$, respectively.

Proof. See Appendix C.2.

Because of the nonlinearity of $\beta_{i}$ in $\Delta_{8}$, it is not easy to obtain a complete geometric characterisation for $L_{1}=2, L_{2}=3$. Hence we rely on numerical simulation in the following subsection to discuss the dynamics.

4.2.2. Dynamics of the Nonlinear System-Numerical Analysis. For $\left(L_{1}, L_{2}\right)=(2,3)$, we choose the set of parameters $\delta_{1}=0.15, \delta_{2}=0.3, \beta=11, b_{1}=b_{2}=0, w=$ $-0.6, a_{1}=0.8, a_{2}=1$. Since $\delta_{1}<1 / 2$, the steady state become unstable through a flip bifurcation. It is found that the price behaviour generated through bifurcation with respect to the parameter $\alpha$ is different from to that through bifurcation with respect to the risk aversion coefficients.

For fixed risk aversion coefficients $A_{1}=A_{2}=0.005$, the price dynamics generated through the bifurcation parameter $\alpha$ are similar to the case of $\left(L_{1}, L_{2}\right)=(1,3)$. That is, as $\alpha$ decreases, the steady state price becomes unstable and prices converge to 2-period cycle, and then to aperiodic cycles (characterised by two coexisting closed orbits), and then to simple periodic cycles again. In addition, the variability of the prices also increases as $\alpha$ decreases.

For fixed $\alpha=-4$, changing of the risk aversion coefficients can generate very rich dynamics. For fixed $A_{1}=0.05$, the bifurcation diagram with respect to the parameter $A_{2}$ is plotted in Figure 4.10. One can see that various types of cycles and strange attractors can be generated as agents become more risk averse.

Instead of $\delta_{1}=0.15<1 / 2$, we can select $\delta_{1}=0.6>1 / 2$. In this case, the steady state price can become unstable through either a flip or Hopf bifurcation. A similar price pattern and bifurcation route to complicated price dynamics can be observed (not shown here) as the risk aversion coefficients change.

\section{Dynamics of the Heterogeneous Model With Infinite Memory GDP}

From the discussion in the previous section, we can see that the lags involved in the GDP can have different effects on the stability of the steady state price and price dynamics. In this section, we consider the limiting case when both lags tend to infinity. 


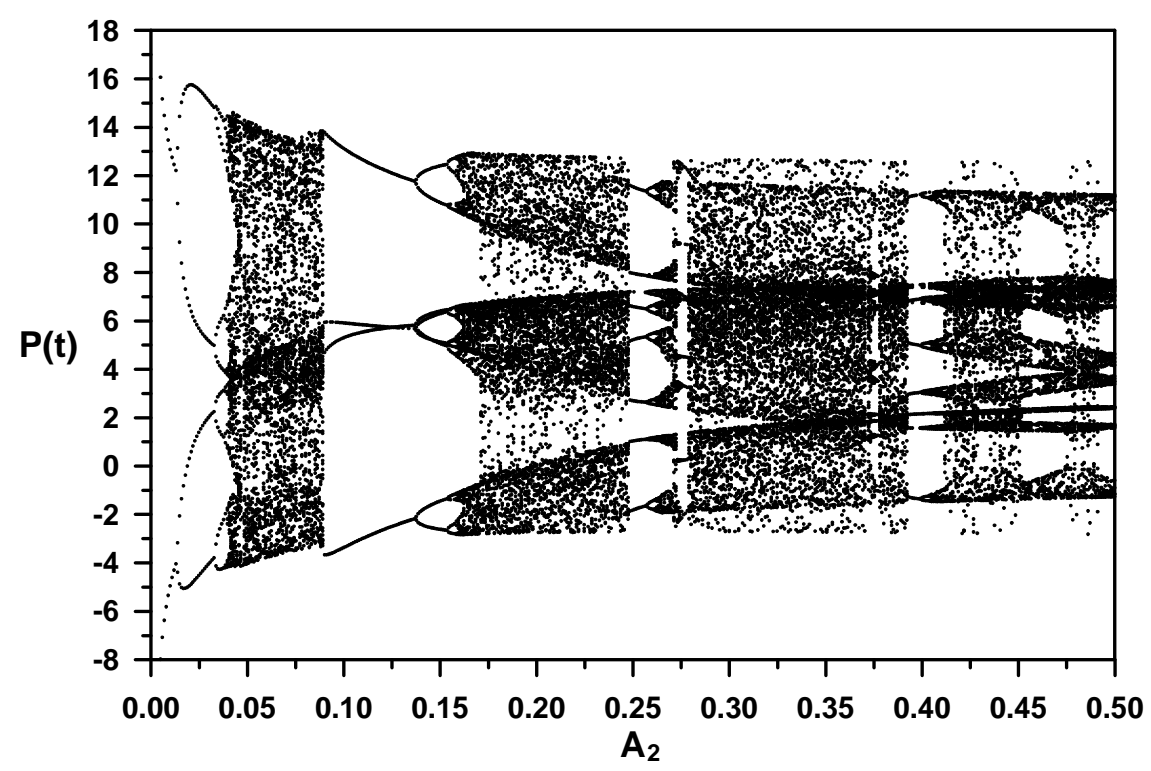

FIGURE 4.10. Bifurcation diagram for parameter $A_{2}$ with parameters $\left(L_{1}, L_{2}\right)=(2,3), A_{1}=0.05, \alpha=-4, w=-0.6, \delta_{1}=0.15, \delta_{2}=$ $0.3, a_{1}=0.8, a_{2}=1, \beta=11, b_{1}=b_{2}=0$.

Let $\delta_{i}$ be the decay rate of agent $i$ 's memory. Then it follows from (2.9) that the dynamics of the conditional mean $m_{i, t}$ and variance $v_{i, t}$ are given by

$$
\left\{\begin{aligned}
m_{1, t} & =\delta_{1} m_{1, t-1}+\left(1-\delta_{1}\right) p_{t-1} \\
m_{2, t} & =\delta_{2} m_{2, t-1}+\left(1-\delta_{2}\right) p_{t-1} \\
v_{1, t} & =\delta_{1} v_{1, t-1}+\delta_{1}\left(1-\delta_{1}\right)\left(p_{t}-m_{1, t-1}\right)^{2} \\
v_{2, t} & =\delta_{2} v_{2, t-1}+\delta_{2}\left(1-\delta_{2}\right)\left(p_{t}-m_{1, t-1}\right)^{2}
\end{aligned}\right.
$$

Let $x_{t}=m_{1, t}, y_{t}=m_{2, t}, z_{t}=v_{1, t}, u_{t}=v_{2, t}$. Then, under the GDP with infinite memory (5.1), the nonlinear cobweb system (2.6) is equivalent to the 5-dimensional system

$$
\left\{\begin{aligned}
p_{t} & =f(p, x, y, z, u)_{t-1} \\
x_{t} & =\delta_{1} x_{t-1}+\left(1-\delta_{1}\right) p_{t-1} \\
y_{t} & =\delta_{2} y_{t-1}+\left(1-\delta_{2}\right) p_{t-1} \\
z_{t} & =\delta_{1} z_{t-1}+\delta_{1}\left(1-\delta_{1}\right)\left(p_{t}-x_{t-1}\right)^{2} \\
u_{t} & =\delta_{2} u_{t-1}+\delta_{2}\left(1-\delta_{2}\right)\left(p_{t}-y_{t-1}\right)^{2}
\end{aligned}\right.
$$

where

$$
f(p, x, y, z, u) \equiv \beta+\frac{\alpha}{2}\left[(1+w) \frac{x-b_{1}}{a_{1}+2 A_{1} z}+(1-w) \frac{y-b_{2}}{a_{2}+2 A_{2} z}\right]
$$

We can state the following result concerning local stability and bifurcation. 
Proposition 5.1. The steady state price $p^{*}$ of the nonlinear cobweb dynamics (2.6) under GDP (5.1) is LAS if

$$
\begin{aligned}
& {\left[\delta_{1} \beta_{2}\left(1-\delta_{2}\right)+\delta_{2} \beta_{1}\left(1-\delta_{1}\right)-\frac{\delta_{1}+\delta_{2}}{2}\right]^{2}} \\
& +\beta_{2}\left(1-\delta_{2}\right)+\beta_{1}\left(1-\delta_{1}\right)<1+\frac{\left(\delta_{1}-\delta_{2}\right)^{2}}{4} .
\end{aligned}
$$

Furthermore, the steady state becomes unstable through a Neimark-Hopf bifurcation. In particular, when $\delta_{1}=\delta_{2}=\delta$, the steady state is stable if $\beta \equiv \beta_{1}+\beta_{2}<1 /(1-\delta)$ and becomes unstable through a Neimark-Hopf bifurcation.

Proof. See Appendix D.

It is interesting to see that, when the memory is infinite, the steady state becomes unstable through a Neimark-Hopf bifurcation only. It may not be easy to see the effect of the decay rates on the stability region from condition (5.3), but the condition when $\delta_{1}=\delta_{2}=\delta$ indicates that the local stability parameter region in terms of $\beta=\beta_{1}+\beta_{2}$ is enlarged as $\delta$ increases, as shown in Figure 5.1(a). In addition, the local stability region becomes unbounded as $\delta \rightarrow 1$. This general feature also holds when $\delta_{1} \neq \delta_{2}$ and this can be verified by numerical plots of the bifurcation surface (not reported here). Hence in general it seems that the stability region is enlarged as the decay rates increase.

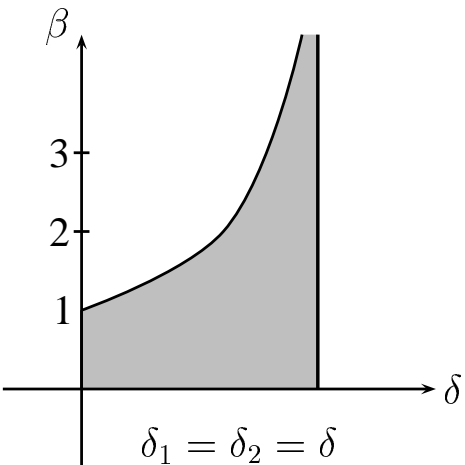

(a)

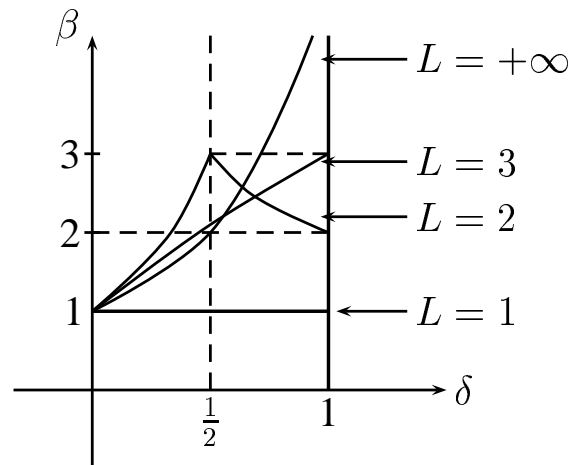

(b)

FIGURE 5.1. (a) Stability region and bifurcation boundary for GDP with $L=\infty$ and $\delta_{1}=\delta_{2}=\delta$; (b) Comparison of stability regions for $L_{1}=L_{2}=L=1,2,3, \infty$ and $\delta_{1}=\delta_{2}=\delta$.

For $\delta_{1}=\delta_{2}=\delta$, a comparison between $L_{1}=L_{2}=L=1,2,3$ and $L=\infty$ is displayed in Figure 5.1(b). ${ }^{8}$ One can see that, for small memory decay rate $\delta$, the stability region may not be enlarged as $L$ increases from finite values to infinity. However, this is indeed the case as the memory decay rate $\delta$ is close to 1 . Therefore, loosely speaking, a high decay rate with long memory can improve the stability of the steady state price.

Numerical simulations can be used to show various price dynamics when the steady state price becomes unstable and indicate that the price dynamics are more dependent

${ }^{8}$ The $L=1,2,3$ cases are reproduced from Figure 4.4(c). 
on the decay rates, rather than the risk aversion coefficients. For the set of parameters: $\beta=11, w=0, a_{1}=0.8, a_{2}=1, b_{1}=b_{2}=0$, we have made the following observations. When both the decay rates are high, say $\delta_{1}=0.6, \delta_{2}=0.9$, the steady state price becomes unstable when the demand slope $\alpha$ is small, say $\alpha=-8$. As $\alpha$ decreases further, prices oscillate quasi-periodically, characterised by closed orbits in the phase plot, with high variability, indicated by Figure 5.2(a). Also, for fixed $\alpha$, a sufficient high $\delta_{i}$ (close to 1) can lead otherwise unstable price dynamics to converge to the steady state price, as indicated by the above local stability analysis.
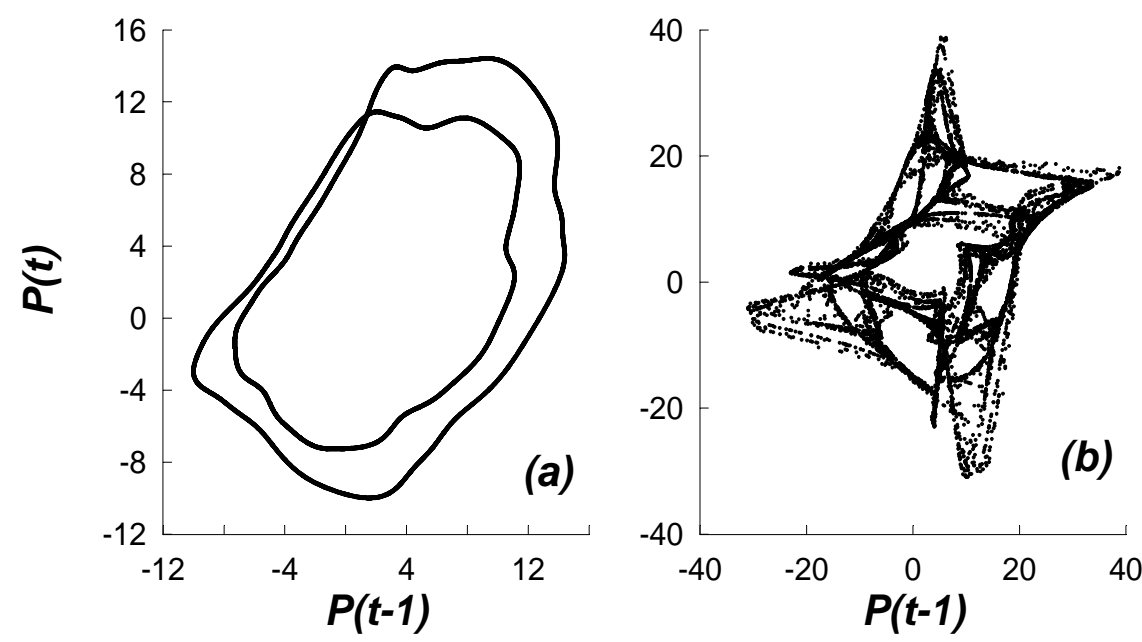

FIGURE 5.2. Phase plot of the nonlinear system for GDP with infinite memory and $A_{1}=A_{2}=0.05, w=0, \delta_{2}=0.9, a_{1}=0.8, a_{2}=1, \beta=$ $11, b_{1}=b_{2}=0$, and (a) $\alpha=-20, \delta_{1}=0.6$; (b) $\alpha=-10, \delta_{1}=0.2$.

For fixed $\alpha=-10, \delta_{1}=0.2, \delta_{2}=0.9$ and $A_{1}=0.05$, prices converge to some strange attractors for a wide range of $A_{2}$ (say $A_{2} \in(0.05,2)$ ), as shown in Figure 5.2(b) for $A_{1}=0.05$. However, for fixed $A_{2}$, say $A_{2}=0.05$, as $A_{1}$ increases from 0.05 up to 2 , prices in the phase plane converge to strange attractors for $A_{1}$ small (say, $\left.A_{1} \in(0.05,0.8)\right)$, and then to a 5-period cycle for $A_{1}=1.2$, and then to a strange attractor for $A_{1}=1.5$. The bifurcation diagram with respect to the parameter $A_{1}$ is plotted in Figure 5.3. This indicates that when agents have infinite memory, the risk aversion coefficient has no significant influence on the price dynamics when agents have a high decay rate (and in particular, when agents have almost full memory over the whole history of prices). However the effect of the risk aversion coefficient can be significant when agents have a low decay rate.

\section{Conclusions}

In this paper we have introduced a heterogeneous GDP learning mechanism into the traditional cobweb model with risk averse heterogeneous agents by allowing producers to learn both mean and variance with different geometric decay rates and different memory lengths. For a class of nonlinear forward-looking models with homogeneous agents, Barucci $(2000,2001)$ shows that, when the memory is infinite, the memory 


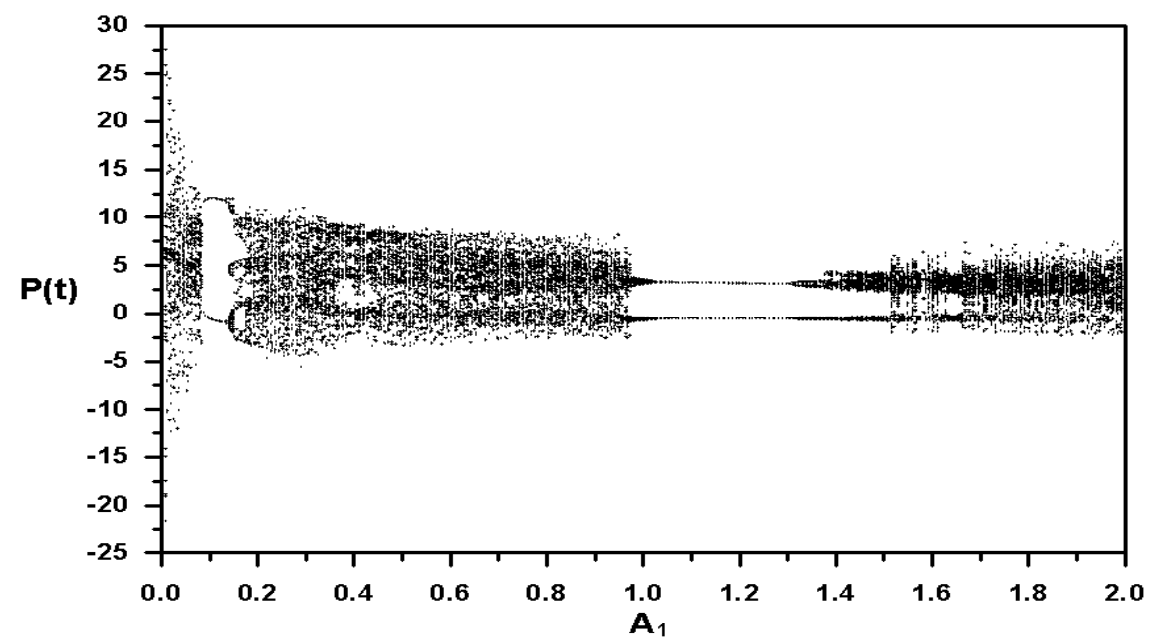

FIGURE 5.3. Bifurcation diagram of the nonlinear system for GDP with infinite memory for parameter $A_{1}$, here $\alpha=-10, \delta_{1}=0.2, \delta_{2}=$ $0.9, A_{2}=0.05, w=0, a_{1}=0.8, a_{2}=1, \beta=11, b_{1}=b_{2}=0$.

decay rate plays a stabilizing role in the sense that increasing the decay rate of the learning process the parameter stability region of a stationary rational expectation equilibrium becomes larger and eliminates cycles and chaotic attractors are created through flip bifurcation, but not Hopf bifurcation. We have shown in this paper that the memory decay rate plays a similar stabilizing role and complicated price dynamics can be created through Neimark-Hopf bifurcation, not flip bifurcation, when memory is infinite and agents are heterogeneous. However, when memory is finite, we show that the decay rate of the GDP of heterogeneous producers plays a complicated role on the price dynamics. When both the lag lengths are odd, increasing the decay rate enlarges the parameters region of the stability of the steady state and complicated price dynamics can only be created through flip bifurcation. However when both the lag lengths are not odd, there exists a critical value (between 0 and 1) such that, when the decay rate is below the critical value, the decay rate plays a stabilizing role and, for the decay rate above the critical value, the decay rate plays a destabilizing role in the sense that the parameter region of local stability of the steady state becomes smaller as the decay rate increases. In addition, (quasi)periodic cycles and strange attractors can be created through flip bifurcations when the decay rate is below the critical value and through Neimark-Hopf bifurcations when the decay rate is above the critical value. It is also found that the source of risk is the risk itself in the sense that the behaviour of producers in response to risk can generate complicated price dynamics and market failure.

The heterogeneous GDP considered in this paper are some of the simplest learning processes and the analysis has shown how they yield very rich dynamics in terms of the stability, bifurcation and routes to complicated dynamics. It is found that the market fractions of heterogeneous agents plays an important role. It would be very interesting to see how the price dynamics are changed when different types of learning schemes (such as naive expectation, ALP and GDP) are competing each other and agents update 
their beliefs based on certain fitness measures, as in Brock and Hommes (1997). In practice, agents revise their expectations by adapting the decay rate in accordance with observations. How the GDP learning affects the dynamics in this more general situation is a question left for future work.

\section{Appendix A. Mean and Variance of GDP with Infinite Memory}

Let $m_{t}$ and $v_{t}$ be the mean and variance of the GDP with lag length $L$, that is

$$
\left\{\begin{aligned}
m_{t-1}= & B\left[p_{t-1}+\delta p_{t-2}+\cdots+\delta^{L-1} p_{t-L}\right] \\
v_{t-1}= & B\left[\left(p_{t-1}-m_{t-1}\right)^{2}+\delta\left(p_{t-2}-m_{t-1}\right)^{2}\right. \\
& \left.+\delta^{2}\left(p_{t-3}-m_{t-1}\right)^{2}+\cdots+\delta^{L-1}\left(p_{t-L}-m_{t-1}\right)^{2}\right]
\end{aligned}\right.
$$

where $B=(1-\delta) /\left(1-\delta^{L}\right)$ for $\delta \in[0,1)$ and $B=1 / L$ for $\delta=1$. The mean process $m_{t}$ can be rearranged as follows:

$$
m_{t}=B\left[p_{t}-\delta^{L} p_{t-L}\right]+\delta m_{t-1}
$$

Then for $\delta \in[0,1)$, as $L \rightarrow \infty$, the limiting mean process is given by

$$
m_{t}=(1-\delta) p_{t}+\delta m_{t-1}
$$

which can be written as

$$
m_{t}-m_{t-1}=(1-\delta)\left(p_{t}-m_{t-1}\right),
$$

or

$$
m_{t}-p_{t}=\delta\left(m_{t-1}-p_{t}\right)
$$

For the variance process, from

$$
v_{t}=B\left[\left(p_{t}-m_{t}\right)^{2}+\delta\left(p_{t-1}-m_{t}\right)^{2}+\cdots+\delta^{L-1}\left(p_{t-(L-1)}-m_{t}\right)^{2}\right],
$$

we have

$$
\begin{aligned}
v_{t}-\delta v_{t-1}= & B\left[\left(p_{t}-m_{t}\right)^{2}+\delta\left[\left(p_{t-1}-m_{t}\right)^{2}-\left(p_{t-1}-m_{t-1}\right)^{2}\right]\right. \\
& +\delta^{2}\left[\left(p_{t-2}-m_{t}\right)^{2}-\left(p_{t-2}-m_{t-1}\right)^{2}\right]+\cdots \\
& +\delta^{L-1}\left[\left(p_{t-(L-1)}-m_{t}\right)^{2}-\left(p_{t-(L-1)}-m_{t-1}\right)^{2}\right]-\delta^{L}\left(p_{t-L}-m_{t-1}\right)^{2},
\end{aligned}
$$

which can be rewritten as

$$
\begin{aligned}
v_{t}-\delta v_{t-1}= & B\left(p_{t}-m_{t}\right)^{2}-B \delta^{L}\left(p_{t-L}-m_{t-1}\right)^{2} \\
& +B\left\{\delta\left[\left(p_{t-1}-m_{t}\right)+\left(p_{t-1}-m_{t-1}\right)\right]\left[m_{t-1}-m_{t}\right]\right. \\
& +\delta^{2}\left[\left(p_{t-2}-m_{t}\right)+\left(p_{t-2}-m_{t-1}\right)\right]\left[m_{t-1}-m_{t}\right]+\cdots \\
& \left.+\delta^{L-1}\left[\left(p_{t-(L-1)}-m_{t}\right)+\left(p_{t-(L-1)}-m_{t-1}\right)\right]\left[m_{t-1}-m_{t}\right]\right\} \\
= & B\left(p_{t}-m_{t}\right)^{2}-B \delta^{L}\left(p_{t-L}-m_{t-1}\right)^{2} \\
& +\left(m_{t-1}-m_{t}\right)\left\{B \left[\delta\left(p_{t-1}-m_{t}\right)+\delta^{2}\left(p_{t-2}-m_{t}\right)\right.\right. \\
& \left.+\cdots+\delta^{L-1}\left(p_{t-(L-1)}-m_{t}\right)\right] \\
& \left.+B\left[\delta\left(p_{t-1}-m_{t-1}\right)+\delta^{2}\left(p_{t-2}-m_{t-1}\right)+\cdots+\delta^{L-1}\left(p_{t-(L-1)}-m_{t}\right)\right]\right\} \\
= & B\left(p_{t}-m_{t}\right)^{2}-B \delta^{L}\left(p_{t-L}-m_{t}\right)^{2} \\
& +\left(m_{t-1}-m_{t}\right)\left[-B\left(p_{t}-m_{t}\right)-B \delta^{L}\left(p_{t-L}-m_{t}\right)\right]
\end{aligned}
$$

Note that, for $\delta \in[0,1)$, as $L \rightarrow \infty, B=(1-\delta) /\left(1-\delta^{L}\right) \rightarrow 1-\delta$ and, using (A.3),

$$
\begin{aligned}
p_{t-L}-m_{t} & =\delta\left(p_{t-L}-m_{t-1}\right)=\delta^{2}\left(p_{t-L}-m_{t-2}\right)=\cdots \\
& =\delta^{L}\left(p_{t-L}-m_{t-L}\right) \rightarrow 0 .
\end{aligned}
$$

Therefore the limiting variance process is given by

$$
\begin{aligned}
v_{t}-\delta v_{t-1} & =(1-\delta)\left(p_{t}-m_{t}\right)^{2}+\left(m_{t-1}-m_{t}\right)\left[-(1-\delta)\left(p_{t}-m_{t}\right)\right] \\
& =(1-\delta)\left(p_{t}-m_{t}\right)\left[\left(p_{t}-m_{t}\right)+\left(m_{t}-m_{t-1}\right)\right] \\
& =(1-\delta)\left(p_{t}-m_{t}\right)\left(p_{t}-m_{t-1}\right)
\end{aligned}
$$

that is,

$$
v_{t}=\delta v_{t-1}+(1-\delta)\left(p_{t}-m_{t}\right)\left(p_{t}-m_{t-1}\right)
$$

Based on the above argument, for $\delta \in[0,1$ ), the limiting process (as $L \rightarrow \infty$ ) of the mean and variance are given by

$$
\left\{\begin{aligned}
m_{t} & =\delta m_{t-1}+(1-\delta) p_{t} \\
v_{t} & =\delta v_{t-1}+(1-\delta)\left(p_{t}-m_{t}\right)\left(p_{t}-m_{t-1}\right) \\
& =\delta v_{t-1}+\delta(1-\delta)\left(p_{t}-m_{t-1}\right)^{2}
\end{aligned}\right.
$$


Appendix B. Characteristic Equation of the Heterogeneous GDP Model with Finite Memory

When the memory is finite, the heterogeneous GDP can be written as

$$
\left\{\begin{aligned}
\bar{p}_{i, t} & =\sum_{j=1}^{L_{i}} w_{i j} p_{t-j}, \\
\bar{v}_{i, t} & =\sum_{j=1}^{L_{i}} w_{i j}\left[\bar{p}_{i, t}-p_{t-j}\right]^{2},
\end{aligned}\right.
$$

in which, $w_{i j}=B_{i} \delta^{j-1}\left(i=1,2\right.$ and $\left.j=1, \cdots, L_{i}\right)$. Let

$$
\left\{\begin{aligned}
x_{1, t} & =p_{t} \\
x_{2, t} & =p_{t-1} \\
x_{3, t} & =p_{t-2} \\
\vdots & \\
x_{L, t} & =p_{t-(L-1)}
\end{aligned}\right.
$$

where $L=\max \left\{L_{1}, L_{2}\right\}$. Then, (2.6) with finite memory GDP is equivalent to the following $L$-dimensional difference system

$$
\left\{\begin{aligned}
x_{1, t+1} & =f\left(x_{t}\right) \\
x_{2, t+1} & =x_{1, t} \\
& \vdots \\
x_{L, t+1} & =x_{L-1, t}
\end{aligned}\right.
$$

where

$$
\begin{cases}f\left(\mathbf{x}_{t}\right) & =\beta+\frac{\alpha}{2}(1+w) \frac{\bar{x}_{1, t}-b_{1}}{a_{1}+2 A_{1} \bar{v}_{1}}+\frac{\alpha}{2}(1-w) \frac{\bar{x}_{2, t}-b_{2}}{a_{2}+2 A_{2} \bar{v}_{2}} \\ \mathbf{x}_{t} & =\left(x_{1, t}, x_{2, t}, \cdots, x_{L, t}\right) \\ \bar{x}_{i, t} & =\sum_{j i}^{L=1} w_{i j} x_{j, t} \\ \bar{v}_{i, t} & =\sum_{j=1}^{L} w_{i j}\left[\bar{x}_{i, t}-x_{j, t}\right]^{2} .\end{cases}
$$

At the steady state $p^{*}, \bar{x}_{1}=\bar{x}_{2}=p^{*}$ and $\bar{v}_{1}=\bar{v}_{2}=0$. Without loss generality, it is assumed that $L_{1} \leq L_{2}$ and then $L=L_{2}$. Evaluating function $f\left(\mathbf{x}_{t}\right)$ at the steady state, one obtains that

$$
\frac{\partial f}{\partial x_{j}}=\frac{\alpha}{2}\left[(1+w) \frac{1}{a_{1}} w_{1 j}+(1-w) \frac{1}{a_{2}} w_{2 j}\right]-\left[w_{1 j} \beta_{1}+w_{2 j} \beta_{2}\right]
$$

for $j=1, \cdots, L_{1}$ and $\frac{\partial f}{\partial x_{j}}=-w_{2 j} \beta_{2}$ for $j=L_{1}+1, \cdots, L$. Therefore the corresponding characteristic equation is given by

$$
\Gamma(\lambda) \equiv \lambda^{L}+\sum_{j=1}^{L_{1}}\left[w_{1 j} \beta_{1}+w_{2 j} \beta_{2}\right] \lambda^{L-j}+\sum_{j=L_{1}+1}^{L} w_{2 j} \beta_{2} \lambda^{L-j} .
$$

In particular, for the GDP, it follows from $w_{i j}=B_{i} \delta^{j-1}$ with $B_{i}=\left(1-\delta_{i}\right) /\left(1-\delta_{i}^{L_{i}}\right), L_{1} \leq L_{2}$ and (B.3) that

$$
\Gamma(\lambda) \equiv \lambda^{L}+\sum_{j=1}^{L_{1}}\left[\beta_{1} B_{1} \delta_{i}^{j-1}+\beta_{2} B_{2} \delta_{2}^{j-1}\right] \lambda^{L-j}+\sum_{j=L_{1}+1}^{L} \beta_{2} B_{2} \delta_{2}^{j-1} \lambda^{L-j}=0 .
$$

\section{Appendix C. Local Stability and Bifurcation Analysis of GDP with Finite Memory}

C.1. The case $L_{1}=L_{2}=L$. When $L_{1}=L_{2}=L$, one can see from (B.4) that the corresponding characteristic equation is given by

$$
\Gamma_{L}(\lambda) \equiv \lambda^{L}+\sum_{j=1}^{L}\left[\beta_{1} B_{1} \delta_{1}^{j-1}+\beta_{2} B_{2} \delta_{2}^{j-1}\right] \lambda^{L-j}=0 .
$$

For $L=1, \Gamma_{1}(\lambda) \equiv \lambda+\left[\beta_{1}+\beta_{2}\right]=0$. Hence, $|\lambda|<1$ holds if and only if $\beta \equiv \beta_{1}+\beta_{2}<1$. Furthermore, $\lambda=-1$ when $\beta=1$, which leads to a flip bifurcation.

For $L=2$, the characteristic equation has the form

$$
\Gamma_{2}(\lambda) \equiv \lambda^{2}+\left[\beta_{1} B_{1}+\beta_{2} B_{2}\right] \lambda+\left[\beta_{1} B_{1} \delta_{1}+\beta_{2} B_{2} \delta_{2}\right]=0,
$$

where $B_{i}=1 /\left(1+\delta_{i}\right)(i=1,2)$. It follows from Jury's test that $\left|\lambda_{i}\right|<1$ if and only if;

(i) $\Gamma_{2}(1)=1+\beta_{1}+\beta_{2}>0$;

(ii) $\Gamma_{2}(-1)=1-\left[\beta_{1} B_{1}+\beta_{2} B_{2}\right]+\left[\beta_{1} B_{1} \delta_{1}+\beta_{2} B_{2} \delta_{2}\right]>0$, which can be rewritten as

$$
\Delta_{2} \equiv \frac{1-\delta_{1}}{1+\delta_{1}} \beta_{1}+\frac{1-\delta_{2}}{1+\delta_{2}} \beta_{2}<1 .
$$


(iii) $\beta_{1} B_{1} \delta_{1}+\beta_{2} B_{2} \delta_{2}<1$, which can be rewritten as

$$
\Delta_{1} \equiv \frac{\delta_{1}}{1+\delta_{1}} \beta_{1}+\frac{\delta_{2}}{1+\delta_{2}} \beta_{2}<1
$$

Therefore, $\left|\lambda_{i}\right|<1$ if and only if (C.2) and (C.3) hold. Note that $\Gamma_{2}(-1)=0$ implies that a flip bifurcation occurs when $\Delta_{2}=1$. Also, when $\lambda_{1,2}=e^{ \pm 2 \pi \theta i}$, we have $\lambda_{1} \lambda_{2}=\beta_{1} B_{1} \delta_{1}+\beta_{2} B_{2} \delta_{2}=\Delta_{1}=1$ and $\lambda_{1}+\lambda_{2}=-\left[\beta_{1} B_{1}+\beta_{2} B_{2}\right]=$ $2 \cos (2 \pi \theta) \equiv \rho$, which implies that $\Delta_{1}=1$ leads to a Neimark-Hopf bifurcation.

When the local stability region is bounded by a Neimark-Hopf bifurcation curve, the nature of the bifurcation is characterised by values of $\rho$, with different regions for different combination of $\left(\delta_{1}, \delta_{2}\right)$.

For $1 / 2 \leq \delta_{1}, \delta_{2} \leq 1$, the stability region is bounded only by the Neimark-Hopf bifurcation boundary $\Delta_{1}=1$. Then, $\rho=-1 / \delta_{2}$ for $\left(\beta_{1}, \beta_{2}\right)=\left(0,\left[1+\delta_{2}\right] / \delta_{2}\right)$ and $\rho=-1 / \delta_{1}$ for $\left(\beta_{1}, \beta_{2}\right)=\left(\left[1+\delta_{1}\right] / \delta_{1}, 0\right)$. Hence

$$
\rho \equiv 2 \cos (2 \pi \theta) \in\left(-\frac{1}{\min \left(\delta_{1}, \delta_{2}\right)}, \quad-\frac{1}{\max \left(\delta_{1}, \delta_{2}\right)}\right) .
$$

For $0 \leq \delta_{1} \leq 1 / 2,1 / 2 \leq \delta_{2} \leq 1$, the stability region is bounded by both flip and Neimark-Hopf bifurcation boundaries. The Neimark-Hopf bifurcation boundary corresponds to the line segment between $A:\left(\beta_{1}, \beta_{2}\right)=\left(0,\left[1+\delta_{2}\right] / \delta_{2}\right)$ and $B$ which is the interaction point between $\Delta_{1}=1$ and $\Delta_{2}=1$, leading to $\rho=-2$. Therefore,

$$
\rho \equiv 2 \cos (2 \pi \theta) \in\left(-2, \quad-\frac{1}{\max \left(\delta_{1}, \delta_{2}\right)}\right) .
$$

For $L=3$, the characteristic equation has the form $\Gamma_{3}(\lambda) \equiv \lambda^{3}+c_{1} \lambda^{2}+c_{2} \lambda+c_{3}=0$, where

$$
\begin{aligned}
& c_{1}=\left[\beta_{1} B_{1}+\beta_{2} B_{2}\right], \quad c_{2}=\left[\beta_{1} B_{1} \delta_{1}+\beta_{2} B_{2} \delta_{2}\right], \\
& c_{3}=\left[\beta_{1} B_{1} \delta_{1}^{2}+\beta_{2} B_{2} \delta_{2}^{2}\right], \quad B_{i}=1 /\left[1+\delta_{i}+\delta_{i}^{2}\right], \quad(i=1,2) .
\end{aligned}
$$

It follows from Jury's test that $\left|\lambda_{i}\right|<1$ if and only if;

(i) $\Gamma_{3}(1)=1+\beta_{1}+\beta_{2}>0$;

(ii) $(-1)^{3} \Gamma_{3}(-1)>0$, which is equivalent to

$$
\Delta_{3} \equiv \frac{1-\delta_{1}+\delta_{1}^{2}}{1+\delta_{1}+\delta_{1}^{2}} \beta_{1}+\frac{1-\delta_{2}+\delta_{2}^{2}}{1+\delta_{2}+\delta_{2}^{2}} \beta_{2}<1
$$

(iii) $c_{2}+c_{3}\left(c_{3}-c_{1}\right)<1$, which is equivalent to

$$
\delta_{1} \gamma_{1}+\delta_{2} \gamma_{2}+\left(\delta_{1}^{2} \gamma_{1}+\delta_{2}^{2} \gamma_{2}\right)\left[\left(\delta_{1}^{2}-1\right) \gamma_{1}+\left(\delta_{2}^{2}-1\right) \gamma_{2}\right]<1,
$$

$$
\text { where } \gamma_{i}=\frac{\beta_{i}}{1+\delta_{i}+\delta_{i}^{2}} \text {. }
$$

(iv) $c_{2} \equiv \delta_{1} \gamma_{1}+\delta_{2} \gamma_{2}<3$

It follows from $\beta_{i}>0, \delta_{i} \in[0,1]$ and $\delta_{i}<1-\delta_{i}+\delta_{i}^{2}$ that condition (i) is satisfied and condition (ii) implies conditions (iii) and (iv). Hence the only condition for $\left|\lambda_{i}\right|<1$ is $\Delta_{3}<1$. In addition, $\lambda=-1$ when $\Delta_{3}=1$, implying that the stability region is bounded by the flip bifurcation boundary defined by $\Delta_{3}=1$.

C.2. The Case $\left(L_{1}, L_{2}\right)=(2,3)$. For $L_{1}=2, L_{2}=3$, the characteristic equation is given by $\Gamma_{2,3}(\lambda) \equiv \lambda^{3}+c_{1} \lambda^{2}+$ $c_{2} \lambda+c_{3}=0$, where

$$
c_{1}=\left[\gamma_{1}+\gamma_{2}\right], \quad c_{2}=\gamma_{1} \delta_{1}+\gamma_{2} \delta_{2}, \quad c_{3}=\gamma_{2} \delta_{2}^{2}, \quad \gamma_{1}=\beta_{1} /\left[1+\delta_{1}\right], \quad \gamma_{2}=\beta_{2} /\left[1+\delta_{2}+\delta_{2}^{2}\right] .
$$

It follows from Jury's test that $\left|\lambda_{i}\right|<1$ if and only if;

(i) $\Gamma_{2,3}(1)=1+\beta_{1}+\beta_{2}>0$;

(ii) $(-1)^{3} \Gamma_{2,3}(-1)>0$, which is equivalent to

$$
\Delta_{7} \equiv \frac{1-\delta_{1}}{1+\delta_{1}} \beta_{1}+\frac{1-\delta_{2}+\delta_{2}^{2}}{1+\delta_{2}+\delta_{2}^{2}} \beta_{2}<1
$$

(iii) $c_{2}+c_{3}\left(c_{3}-c_{1}\right)<1$, which is equivalent to

$$
\Delta_{8} \equiv \frac{\delta_{1}}{1+\delta_{1}} \beta_{1}+\frac{\delta_{2}}{1+\delta_{2}+\delta_{2}^{2}} \beta_{2}-\frac{\delta_{2} \beta_{2}}{1+\delta_{2}+\delta_{2}^{2}}\left(\frac{\beta_{1}}{1+\delta_{1}}+\frac{\left(1-\delta_{2}^{2}\right) \beta_{2}}{1+\delta_{2}+\delta_{2}^{2}}\right)<1 .
$$

(iv) $c_{2} \equiv \delta_{1} \gamma_{1}+\delta_{2} \gamma_{2}<3$.

Note that since $\beta_{i}>0, \delta_{i} \in[0,1]$ and $\delta_{2}<1-\delta_{2}+\delta_{2}^{2}$, one can see that $\Delta_{7}<1$ implies condition (iv). In addition $\lambda=-1$ when $\Delta_{7}=1$ is satisfied and $\Delta_{7}<1$ implies $\Delta_{8}<1$ for $\delta_{1} \leq 1 / 2$. 
Appendix D. Proof OF Proposition 5.1

Consider the system

$$
\left\{\begin{aligned}
p_{t} & =f_{1}(p, x, y, z, u)_{t-1} \\
x_{t} & =\delta_{1} x_{t-1}+\left(1-\delta_{1}\right) p_{t-1} \equiv f_{2}, \\
y_{t} & =\delta_{2} y_{t-1}+\left(1-\delta_{2}\right) p_{t-1} \equiv f_{3}, \\
z_{t} & =\delta_{1} z_{t-1}+\delta_{1}\left(1-\delta_{1}\right)\left(p_{t}-x_{t-1}\right)^{2} \equiv f_{4} \\
u_{t} & =\delta_{2} u_{t-1}+\delta_{2}\left(1-\delta_{2}\right)\left(p_{t}-y_{t-1}\right)^{2} \equiv f_{5}
\end{aligned}\right.
$$

with

$$
f_{1} \equiv \beta+\frac{\alpha}{2}\left[(1+w) \frac{x-b_{1}}{a_{1}+2 A_{1} z}+(1-w) \frac{y-b_{2}}{a_{2}+2 A_{2} z}\right] .
$$

At the unique fixed point $\left(p_{t}, x_{t}, y_{t}, z_{t}, u_{t}\right)=\left(p^{*}, p^{*}, p^{*}, 0,0\right)$ evaluate:

$$
\left\{\begin{array}{l}
\frac{\partial f_{1}}{\partial p}=0 \\
\frac{\partial f_{1}}{\partial x}=\frac{\alpha}{2} \frac{(1+w)}{a_{1}}=-\beta_{1}, \\
\frac{\partial f_{1}}{\partial y}=\frac{\alpha}{2} \frac{(1-w)}{a_{2}}=-\beta_{2}, \\
\frac{\partial f_{1}}{\partial z}=\frac{\alpha}{2}(1+w) \frac{-2 A_{1}\left(p^{*}-b_{1}\right)}{a_{1}^{2}} \equiv \Delta_{1}^{*} \\
\frac{\partial f_{1}}{\partial u}=\frac{\alpha}{2}(1+w) \frac{-2 A_{2}\left(p^{*}-b_{2}\right)}{a_{2}^{2}} \equiv \Delta_{2}^{*}
\end{array}\right.
$$

and

$$
\left\{\begin{array}{l}
\frac{\partial f_{2}}{\partial p}=1-\delta_{1}, \quad \frac{\partial f_{2}}{\partial x}=\delta_{1}, \quad \frac{\partial f_{2}}{\partial y}=\frac{\partial f_{2}}{\partial z}=\frac{\partial f}{\partial u}=0 \\
\frac{\partial f_{3}}{\partial p}=1-\delta_{2}, \quad \frac{\partial f_{3}}{\partial y}=\delta_{2}, \quad \frac{\partial f_{3}}{\partial x}=\frac{\partial f_{3}}{\partial z}=\frac{\partial f_{3}}{\partial u}=0 \\
\frac{\partial f_{4}}{\partial p}=0=\frac{\partial f_{4}}{\partial x}=\frac{\partial f_{4}}{\partial y}=0, \quad \frac{\partial f_{4}}{\partial z}=\delta_{1}, \quad \frac{\partial f_{4}}{\partial u}=0 \\
\frac{\partial f_{5}}{\partial u}=\delta_{2}, \quad \frac{\partial f_{5}}{\partial p}=\frac{\partial f_{5}}{\partial x}=\frac{\partial f_{5}}{\partial y}=\frac{\partial f_{5}}{\partial z}=0 .
\end{array}\right.
$$

The Jacobian matrix $J$ is then given by

$$
J=\left[\begin{array}{ccccc}
0 & -\beta_{1} & -\beta_{2} & \Delta_{1}^{*} & \Delta_{2}^{*} \\
1-\delta_{1} & \delta_{1} & 0 & 0 & 0 \\
1-\delta_{2} & 0 & \delta_{2} & 0 & 0 \\
0 & 0 & 0 & \delta_{1} & 0 \\
0 & 0 & 0 & 0 & \delta_{2}
\end{array}\right]
$$

Thus the characteristic equation is given by $\Gamma(\lambda) \equiv|\lambda I-J|=\left(\lambda-\delta_{1}\right)\left(\lambda-\delta_{2}\right) h(\lambda)$, where $h(\lambda)=\lambda^{3}+c_{1} \lambda^{2}+c_{2} \lambda+c_{3}$ and $c_{1}=-\left(\delta_{1}+\delta_{2}\right), c_{2}=\delta_{1} \delta_{2}+\beta_{2}\left(1-\delta_{2}\right)+\beta_{1}\left(1-\delta_{1}\right)$ and $c_{3}=-\delta_{1} \beta_{2}\left(1-\delta_{2}\right)-\delta_{2} \beta_{1}\left(1-\delta_{1}\right)$. For $\delta_{1}, \delta_{2} \in(0,1)$, applying Jury's test to $h(\lambda)=0$, one can see that $\left|\lambda_{i}\right|<1$ if and only if $\pi_{i}>0$, where

$$
\left\{\begin{aligned}
\pi_{1} & =1+c_{1}+c_{2}+c_{3} \\
\pi_{2} & =1-c_{1}+c_{2}-c_{3} \\
\pi_{3} & =1-c_{2}+c_{3}\left(c_{1}-c_{3}\right) \\
c_{2} & <3
\end{aligned}\right.
$$

Note that

$$
\begin{aligned}
\pi_{1}>0 \Leftrightarrow & \left(1-\delta_{1}\right)\left(1-\delta_{2}\right)\left[1+\beta_{1}+\beta_{2}\right]>0, \\
\pi_{2}>0 \Leftrightarrow & -\left[\frac{1-\delta_{1}}{1+\delta} \beta_{1}+\frac{1-\delta_{2}}{1+\delta_{2}} \beta_{2}\right]<1, \\
\pi_{3}>0 \Leftrightarrow & {\left[\delta_{1} \beta_{2}\left(1-\delta_{2}\right)+\delta_{2} \beta_{1}\left(1-\delta_{1}\right)+\frac{\delta_{1}+\delta_{2}}{2}\right]^{2} } \\
& +\beta_{2}\left(1-\delta_{2}\right)+\beta_{1}\left(1-\delta_{1}\right)<1+\frac{\left(\delta_{1}-\delta_{2}\right)^{2}}{4},
\end{aligned}
$$

and $c_{2}<3$ is implied by $\pi_{3}>0$. Therefore, the only condition we need for local stability is $\pi_{3}>0$. Furthermore, from $h(1)=\pi_{1},(-1)^{3} h(-1)=\pi_{2}$, we conclude that there is no saddle-node and flip bifurcation and the only boundary of the stability region is given by Neimark-Hopf bifurcation boundary, defined by $\pi_{3}=0$. Along the bifurcation boundary, let $\lambda_{1,2}=e^{ \pm 2 \pi \theta i}, \lambda_{3}=r \in(-1,1)$. Then it follows from

$$
\begin{aligned}
& {\left[\lambda_{1}+\lambda_{2}+\lambda_{2}\right]=-[\rho+r]=-\left[\delta_{1}+\delta_{2}\right],} \\
& \lambda_{1} \lambda_{2}+\lambda_{1} \lambda_{3}+\lambda_{2} \lambda_{3}=1+r \rho \\
& \quad=\delta_{1} \delta_{2}+\beta_{1}\left(1-\delta_{1}\right)+\beta_{2}\left(1-\delta_{2}\right), \\
& \lambda_{1} \lambda_{2} \lambda_{3}=-r=-\left[\delta_{1} \beta_{2}\left(1-\delta_{2}+\delta_{2} \beta_{1}\left(1-\delta_{1}\right)\right],\right.
\end{aligned}
$$

that $\rho=\delta_{1}\left[1-\beta_{2}\left(1-\delta_{2}\right)\right]+\delta_{2}\left[1-\beta_{1}\left(1-\delta_{1}\right)\right]$. In particular, for $\delta_{1}=\delta_{2}=\delta$, the stability condition becomes $[1-\beta(1-$ $\delta)]\left[\delta^{2} \beta(1-\delta)+\left(1-\delta^{2}\right)\right]>0$, which is equivalent to $\beta<1 /(1-\delta)$, where $\beta=\beta_{1}+\beta_{2}$. Along the bifurcation boundary, $\beta(1-\delta)=1$, and hence $\rho=\delta$. 


\section{REFERENCES}

Artstein, Z. (1983), 'Irregular cobweb dynamics', Economics Letters 11, 15-17.

Balasko, Y. and Royer, D. (1996), 'Stability of competitive equilibrium with respect to recursive and learning processes', Journal of Economic Theory 68, 319-348.

Barucci, E. (2000), 'Exponentially fading memory learning in forward-looking economic models', Journal of Economic Dynamics and Control 24, 1027-1046.

Barucci, E. (2001), 'Fading memory learning in a class of forward-looking models with an application to hyperinflation dynamics', Economic Modelling 18, 233-252.

Bouassard, J. and Gerard, F. (1991), Risk aversion and chaotic motion in agriculatural markets, in 'the FUR VI Conference on Risk and Utility', Cachan, France.

Boussard, J.-M. (1996), 'When risk generates chaos', Journal of Economic Behavior and Organization 29, 433-446.

Branch, W. (2002), 'Local convergence properties of a cobweb model with rationally heterogeneous expectations', Journal of Economic Dynamics and Control 27, 63-85.

Bray, M. (1982), 'Learning, estimation and the stability of rational expectations', Journal of Economic Theory 26, 318-339.

Bray, M. (1983), Convergence to rational expectations equilibria, Cambridge Univ. Press, Cambridge, UK. in Individual Forecasting and Aggregate Outcomes, Eds. R. Frydman and E.S. Phelps.

Brock, W. and Hommes, C. (1997), 'A rational route to randomness', Econometrica 65, 1059-1095.

Burton, M. (1993), 'Some illustrations of chaos in commodity models', Journal of Agricultural Economics 44(1), 38-50.

Chiarella, C. (1988), 'The cobweb model, its instability and the onset of chaos', Economic Modelling 5, 377-384.

Chiarella, C. and He, X. (2000), The Dynamics of the Cobweb when Producers are Risk Averse Learners, Physica-Verlag, pp. 86-100. in Optimization, Dynamics, and Economic Analysis, E.J. Dockner, R.F. Hartl, M. Luptacik and G. Sorger (Eds).

Chiarella, C. and He, X. (2003a), 'Dynamics of beliefs and learning under $\mathbf{a}_{l}$-processes - the heterogeneous case', Journal of Economic Dynamics and Control 27, 503-531.

Chiarella, C. and He, X. (2003b), Economic Complexity: Non-linear Dynamics, Multi-Agents Economies, and Learning, Vol. 14, Paper 15 of ISETE (International Symposium in Economic Theory and Econometrics) Series, Elsevier, Cambridge University Press, chapter Dynamics of Beliefs and Learning under $a_{L}$-Processes - The Homogeneous Case.

Chiarella, C., He, X. and Zhu, P. (2003), 'Fading memory learning in the cobweb model with risk averse heterogeneous producers', School of Finance and Economics, University of Techonology Sydney . Working Paper.

Day, R. (1992), 'Complex economic dynamics: obvious in history, generic in theory, elusive in data', Journal of Applied Econometrics 7, S9-S23.

Day, R. and Huang, W. (1990), 'Bulls, bears and market sheep', Journal of Economic Behavior and Organization 14, 299-329.

Evans, G. and Honkapohja, S. (1994), 'On the local stability of sunspot equilibria under adaptive learning rules', Journal of Economic Theory 64, 142-161.

Evans, G. and Honkapohja, S. (1995), Increasing social returns, learning, and bifurcation phenomena, Blackwell, Oxford. in Learning and Rationality in Economics, Eds. A. Kirman and M. Salmon.

Evans, G. and Honkapohja, S. (1999), Learning Dynamics, chapter 7, pp. 449-542. in Handbook of Macroeconomics, Eds. J.B. Taylor and M. Woodford.

Evans, G. and Ramey, G. (1992), 'Expectation calculation and macroeconomics dynamics', American Economic Review 82, 207-224.

Hale, J. and Kocak, H. (1991), Dynamics and bifurcations, Vol. 3 of Texts in Applied Mathematics, Springer-Verlag, New York.

Holmes, J. and Manning, R. (1988), 'Memory and market stability, the case of the cobweb', Economics Letters 28, 1-7.

Hommes, C. (1991), 'Adaptive learning and roads to chaos: The case of the cobweb', Economics Letters 1991, 127-132. 
Hommes, C. (1994), 'Dynamics of the cobweb model with adaptive expectations and nonlinear supply and demand', Journal of Economic Behavior and Organization 24, 315-335.

Hommes, C. (1998), 'On the consistency of backward-looking expectations: The case of the cobweb', Journal of Economic Behavior and Organization 33, 333-362.

Jensen, R. and Urban, R. (1984), 'Chaotic price behaviour in a nonlinear cobweb model', Economics Letters 15, 235-240.

Kuznetsov, Y. (1995), Elements of applied bifurcation theory, Vol. 112 of Applied mathematical sciences, SV, New York.

Onozaki, T., Sieg, G. and Yokoo, M. (2000), 'Complex dynamics in a cobweb model with adaptive production adjustment', Journal of Economic Behavior and Organization 41, 101-115.

Onozaki, T., Sieg, G. and Yokoo, M. (2003), 'Stability, chaos and multiple attractors: a single agent makes a difference', Journal of Economic Dynamics and Control 27, 1917-1938.

Puu, T. (1991), Nonlinear economic dynamics, Springer Verlag, Berlin.

Sonis, M. (2000), 'Critical bifurcation surfaces of 3d discrete dynamics', Discrete Dynamics in Nature and Society 4, 333-343. 